\title{
Simulation of Liquid Jet Primary Breakup in a Supersonic Crossflow under Adaptive Mesh Refinement Framework
}

\author{
Nan Liu ${ }^{\mathrm{a}}$, Zhenguo Wang ${ }^{\mathrm{a}}$, Mingbo Sun ${ }^{\mathrm{a}, *}$, Ralf Deiterding ${ }^{\mathrm{b}}$, Hongbo Wang ${ }^{\mathrm{a}}$ \\ ${ }^{a}$ Science and Technology on Scramjet Laboratory, National University of Defense \\ Technology, Changsha, 410073, China, \\ ${ }^{\mathrm{b}}$ Aerodynamics and Flight Mechanics Research Group, University of Southampton, \\ Highfield Campus, Southampton SO171BJ, United Kingdom
}

\begin{abstract}
Compressible two-phase flows were simulated based on the five-equation model under the Adaptive Mesh Refinement (AMR) framework to balance the requirements between space resolution and computational cost. And the simulation system was established in an open source software AMROC (Adaptive Mesh Refinement Object-oriented $\mathrm{C}++$ ). A combination of Godunov method and wave propagation method was introduced to integrate numerical methods with the AMR algorithm. High speed and high liquid-gas density ratio are two main challenges in the simulation of liquid jet in a supersonic crossflow. To enhance the robustness of the simulation system, a MOON-type positivity preserving method was adopted in the development of the codes. Based on the system mentioned above, a liquid jet in a Mach 1.5 supersonic crossflow was simulated as the standard case to study the primary

\footnotetext{
*corresponding author

sunmingbo@nudt.edu.cn (Mingbo Sun)
} 
breakup process in the near field. The simulation captured the column and surface breakup which were the results of the development of the unstable waves in two directions respectively. The instabilities causing the surface breakup were found to be generated in the transonic region initially. Crossflow of a higher Mach number (Ma 1.8) was found being able to augment the instable waves along the injection direction and increase the number of instabilities responsible for the surface breakup. While there was no obvious enhancement of the penetration in the condition of periodic injection, extra unstable waves were imposed on both of windward and leeward liquid surface. The introduced unstable waves had an improvement on the column and surface breakup.

Keywords: compressible two-phase model; adaptive mesh refinement; liquid jet; primary breakup; supersonic crossflow

\section{Introduction}

Liquid and gaseous jet in the supersonic crossflows are important research topics in the development of the scramjet or ramjet engine[1,2]. Fuel injected from the orifices on the wall of the combustion chamber experiences complicate processes such as strong shear, interaction with shock wave, strong convection and vortex motion[2]. Although gaseous jet in a supersonic crossflow has been discussed widely[3, 4], liquid fuel is always a practical choice because of its unique advantages in storage and energy density. The mixing between liquid fuel and gas has a direct influence on the ignition[5] and 
combustion efficiency [6-8]. A deep understanding of the liquid fuel breakup process can lead to a promotion of the engine performance. The whole atomization process includes primary breakup and secondary breakup. The primary breakup happens near the injector nozzle where the main behavior is the surface instabilities on the liquid column. And the secondary breakup occurs at the downstream where liquid ligaments or large droplets breakup into smaller droplets.

A large number of experiments[2,9, 10] have been carried out to study the interaction between transverse liquid jet and high-speed gaseous flows in the past. In the last several years, the researches were mainly focused on the liquid jet penetration height[11], liquid jet breakup processes[9], and spray mixing[10]. Capturing optical images is the most direct way to study the liquid jet breakup in a supersonic crossflow. High-speed photography, shadowgraph method and holography method were widely applied in the previous studies on primary breakup of liquid jet in a crossflow. Surface instabilities are the most important and significant phenomenon on liquid jet primary breakup in the near field. In the earlier times, the wave phenomenon was observed by Schetz et al.[12] in liquid jet breakup in a supersonic crossflow and the physical process was suggested to related with surface tension, acceleration, Kelvin-Helmholtz instability or eddies due to turbulence[2, 9, 13, 14]. Sallam et al.[13] proposed that the relationship between wavelength and Weber number is consistent with Rayleigh-Taylor instability, while the results of the experiment by Arienti and Soteriou conversely supported that Kelvin-Helmholtz instability was a better interpretation[14]. And recently, Li found that the dominant mechanism varied from Rayleigh-Taylor instability 
to Kelvin-Helmholtz instability with reducing density ratio[15]. The primary breakup mechanism was mostly studied in the conditions of a subsonic crossflow because the primary breakup region was always obscured by the dense clouds of small droplet striped from the liquid column. $\mathrm{Wu}[9]$ captured the structures of the liquid jet in supersonic crossflows. However, the experimental results still cannot provide enough data on liquid jet primary breakup in a supersonic crossflow and a numerical simulation would be a good supplementary to the experimental studies. To enhance the atomization efficiency, different injection methods were studied widely. Sapmaz et al.[11] studied the pulsed liquid jet with frequency ranging from $1 \mathrm{~Hz}$ to $1000 \mathrm{~Hz}$ and corresponding Strouhal number less than 0.017 . In their research, the pulsing frequency had no significant effect on the penetration height of the liquid jet in Mach 1.5 crossflow. The Strouhal (Str) number higher than 0.2 with frequency around $10 \mathrm{kHz}$ was suggested by Sapmaz et al.[11] to see an obvious effect of the pulsed liquid jet. Pulsed fuel injection studied by Chen and Zhao[16] reach the similar conclusion. Although a large number of experimental works have revealed some important phenomena in the liquid jet breakup process, detailed information is required to reach a deeper understanding of the physical mechanism of liquid jet breakup in supersonic crossflows.

Numerical study can obtain more flow states of interest than the experiments, while accuracy of the numerical results is highly dependent on the physical models and numerical method applied in the simulations. There are two branches in the two-phase simulation: Euler-Lagrange and Euler-Euler systems. The secondary breakup region comprises of dense groups of small liquid droplets. While the interfaces behavior in this 
region is hard to be captured in Eulerian methods, a more efficient way is to track the small droplets in a Lagrangian method. In practice, the gas crossflows are controlled by a set of Eulerian governing equations while the liquid jet is replaced with groups of the small particles. Betelin et al. [17] developed a two-phase simulation system under Euler-Lagrange framework combined with the evaporation and combustion models in addition to the breakup model. And $\operatorname{Li}[18]$ captured the liquid trailing phenomenon in supersonic crossflows with Euler-Lagrange system. Although Euler-Lagrange system provides a practical way to simulate the two-phase flows in a large space scale with limited computational resources, the detailed behaviors cannot be captured in the nearfield region where primary breakup happens. To study the primary breakup mechanism, a better choice would be the Euler-Euler system in which the liquid and gas flows are both solved by the Euler system. The main subject of the two-phase problem in EulerEuler system is the representation of the interfaces among different fluids. Sharp interface method and diffused interface method are two major models. Sharp interface method also known as interface tracking method is an explicit method in which the position of the interface is represented by certain variables, such as front tracking method[19], volume of fluid method[20] and level set method[21]. Zhu[22] applied Coupled Level Set and Volume of Fluid (CLSVOF) method to study a sinusoidal periodic liquid jet in a crossflow in the condition of a low Weber number. However, the interface tracking method would encounter the severe robustness problems in some extreme simulation conditions. At the cost of the interface resolution, the robustness can be guaranteed in the diffused interface method including $\gamma$-based model[23], five- 
equation model[24], effective-fluid model[25] and homogeneous-equilibrium model[26]. To overcome the drawback of the diffused interface method, there are two ways to sharpen the interface: one is to rebuild the distribution of the variables at interfaces mathematically and the other is enhancing the grid resolution. THINC scheme[27] and anti-diffusion method[28] are two kinds of numerical technique to control the development of numerical diffusion which is more obvious in high-speed conditions than low-speed conditions. The primary problem in these two kinds of methods is the conservation. Enhancement of the grid resolution is the most direct way to obtain a sharper interface. The non-uniform grid is proper for simulation of coaxial jets in which the direction of the main flows is accord with the grid coordinate. However, the situation of the jet in crossflows is more complicate. AMR (Adaptive Mesh Refinement) algorithm[29] can provide the high grid resolution and at the same time settle down the unbalance working load in the simulation of the liquid jet in a supersonic crossflow.

In this paper, a five-equation two-phase model was applied in the framework of the ARMOC (Adaptive Mesh Refinement Object-oriented C++)[30, 31]. And a Godunov method was combined with wave propagation method to solve the governing equations. The subject of this paper was to simulate the primary breakup of the liquid jet in a supersonic crossflow under different injection conditions. Following are how this paper organized. The section 2 briefly introduced the physical model used in the simulation. And then, the section 3 presented the numerical methods including HLLC method, wave propagation method, high order variable reconstruction, positivity 
preserving method and AMR algorithm. In the Section 4, a detailed demonstration and discussion of liquid jet breakup in supersonic crossflows were carried out. In the last, a summary of the numerical study was drawn in section 5 .

\section{Physical modelling}

\subsection{Governing equations}

Following our previous work[32], a five-equation model was introduced to simulate compressible two-phase flows with large density difference. In the nondimensionalized governing equations, the surface tension and viscous force are nondimensionalized by the We number and Re number respectively. In the physical problem we concerned, the We number and Re number are very large. As a result, the influence of aerodynamic force is more obvious than the surface tension and viscous force. Therefore, the main subject of our paper was focused on the behavior of the aerodynamic force in the liquid jet primary breakup in a supersonic crossflow. Besides, the five-equation model is the diffused interface method, therefore the molecular viscosity will be covered by numerical viscosity when an extremely high resolution is not reached $[32,33]$. And the system is given below:

$$
\begin{gathered}
\frac{\partial}{\partial t}\left(\alpha_{1} \rho_{1}\right)+\frac{\partial}{\partial x}\left(\alpha_{1} \rho_{1} u\right)=0 \\
\frac{\partial}{\partial t}\left(\alpha_{2} \rho_{2}\right)+\frac{\partial}{\partial x}\left(\alpha_{2} \rho_{2} u\right)=0 \\
\frac{\partial}{\partial t}(\rho u)+\frac{\partial}{\partial x}\left(\rho u^{2}+p\right)=0 \\
\frac{\partial}{\partial t}(\rho E)+\frac{\partial}{\partial x}((\rho E+p) u)=0 \\
\frac{\partial}{\partial t}\left(\alpha_{1}\right)+u \frac{\partial}{\partial x}\left(\alpha_{1}\right)=0
\end{gathered}
$$


Where $\alpha, \rho, u, E$ are volume fraction, density, velocity and specific total energy respectively. The subscripts 1 and 2 denote different phases.

\subsection{Equation of state and mixture rules}

There are various thermodynamic relationships to close the governing equations. And the stiffened gas EOS (Equation of State) was widely applied in many works[34, 35]. The relationship between pressure, density and specific internal energy is given below:

$$
p=\rho \varepsilon(\gamma-1)-\gamma p_{\infty}
$$

Where $\varepsilon$ is the specific internal energy while $\gamma$ and $p_{\infty}$ are constant parameters which represent fluid properties. And the corresponding sonic speed is given as

$$
c=\sqrt{\frac{\gamma\left(p+p_{\infty}\right)}{\rho}}
$$

Because the main subject of this paper is to simulate the liquid jet in supersonic crossflows. Water and air were adopted to represent the liquid and gas, and the parameters in the stiffened gas EOS were taken as Table 1 shows.

Table 1 properties of water and air in stiffened gas EOS

\begin{tabular}{c|cc}
\hline & $\gamma$ & $p_{\infty}$ \\
\hline Air & 1.4 & 0 \\
water & 6.12 & $3.43 \times 10^{8}$ \\
\hline
\end{tabular}

As mentioned in the[32,36], the mixture rule for $\gamma$ and $\mathrm{p}_{\infty}$ are given as

$$
\begin{gathered}
\alpha_{1} \Gamma_{1}+\alpha_{2} \Gamma_{2}=\Gamma \\
\alpha_{1} \Pi_{1}+\alpha_{2} \Pi_{2}=\Pi
\end{gathered}
$$


Where $\Gamma=1 /(\gamma-1)$ and $\Pi=\gamma p_{\infty} /(\gamma-1)$

\section{Numerucal methods}

\subsection{Spatial discretization}

Various numerical methods were utilized to solve the governing equations of compressible multi-phase model, such as AUSM family methods[37] and HLL family methods[38]. Recent years, a new method called Wave Propagation Method (WPM) was developed by Le Veque[39] for hyperbolic problems. Though this method is based on the viewpoint of propagation of discontinuity waves, wave propagation method is equivalent to the Godunov method in solving the conservative equations. As equation (5) shows, there is a non-conservative equation in the five-model (also in other compressible multiphase models). Johnsen and Colonius[23] reformed the advection equation mathematically into a different one in order to adapt the HLLC solver to equation (5), and this form worked well also in $[32,36]$. While this form is not suitable in the framework of Adaptive Mesh Refinement (AMR) originally proposed by Berger and Le Veque[40], the wave propagation method is particularly tailored for advection equation. Therefore, a combination of the Godunov method and the wave propagation method was adopted in this paper. For the equations (1)-(4), the Godunov method was applied and the HLLC Riemann solver returned the fluxes at the cell interfaces. And as presented in the[23], the HLLC flux can be written as

$$
\vec{F}_{i+1 / 2}=\frac{1+\operatorname{sign}\left(\mathrm{s}^{*}\right)}{2}\left[\vec{F}_{L}+\mathrm{s}^{-}\left(\overrightarrow{\mathrm{q}}_{L}^{*}-\overrightarrow{\mathrm{q}}_{L}\right)\right]+\frac{1-\operatorname{sign}\left(\mathrm{s}^{*}\right)}{2}\left[\vec{F}_{R}+\mathrm{s}^{+}\left(\overrightarrow{\mathrm{q}}_{R}^{*}-\overrightarrow{\mathrm{q}}_{R}\right)\right]
$$

Where the superscript * denotes the intermediate state in HLLC, and subscript L/R 
denotes the left/right state at grid point $i+1 / 2$

$$
\begin{gathered}
\vec{q}_{L / R}^{*}=\frac{s_{L / R}-u_{L / R}}{s_{L / R}-s^{*}}\left(\begin{array}{c}
\rho_{L / R} \\
\rho_{L / R} s^{*} \\
E_{L / R}+\left(s^{*}-u_{L / R}\right)\left(\rho_{L / R} s^{*}+\frac{p_{L / R}}{s_{L / R}-u_{L / R}}\right)
\end{array}\right) \\
\chi_{L / R}^{*}=\frac{s_{L / R}-u_{L / R}}{s_{L / R}-s^{*}} \\
s^{*}=\frac{p_{R}-p_{L}+\rho_{L} u_{L}\left(\mathrm{~s}_{L}-\mathrm{u}_{L}\right)-\rho_{R} u_{R}\left(\mathrm{~s}_{R}-\mathrm{u}_{R}\right)}{\rho_{L}\left(\mathrm{~s}_{L}-\mathrm{u}_{L}\right)-\rho_{R}\left(\mathrm{~s}_{R}-\mathrm{u}_{R}\right)}
\end{gathered}
$$

And the wave speeds are given as

$$
s^{-}=\min \left(0, s_{L}\right), s^{+}=\min \left(0, s_{R}\right)
$$

Where $s_{L}=\min \left(\tilde{\mathrm{u}}-\bar{c}, \mathrm{u}_{L}-c_{L}\right), s_{R}=\min \left(\tilde{\mathrm{u}}+\bar{c}, \mathrm{u}_{R}+c_{R}\right), \bar{c}=\min \left(c_{L}, c_{R}\right)$

Where the superscript “ ” denotes the ROE average. Meanwhile, the equation (5), the advection equation, was solved with the wave propagation method. The expression is given below

$$
\alpha_{1, i}^{n+1}-\alpha_{1, i}{ }^{n}=-\frac{\Delta t}{\Delta x}\left(A^{+} \Delta \alpha_{1, i-1 / 2}+A^{-} \Delta \alpha_{1, i+1 / 2}\right)
$$

Where $A^{+}=\max \left(s^{*}{ }_{i-1 / 2}, 0\right), A^{-}=\min \left(s^{*}{ }_{i+1 / 2}, 0\right)$

\subsection{Variable reconstruction}

Following the work $[32,36]$, the characteristic variables projected from the primitive variables are supposed to be reconstructed in case of the possible oscillations at the contact discontinuities when conservative variables are reconstructed. And the variable reconstruction for reaching higher order accuracy introduces extra discontinuities in the wave propagation method. Therefore, a substitutive form of the wave propagation method by Ketcheson[41] was applied in this paper because of the 
introduction of the high order variable reconstruction. And the new form is showed

$$
\alpha_{1, i}^{n+1}-\alpha_{1, i}^{n}=-\frac{\Delta t}{\Delta x}\left(A^{+} \Delta \alpha_{1, i-1 / 2}+A^{-} \Delta \alpha_{1, i+1 / 2}+A\left(\alpha_{1, i+1 / 2}^{L}-\alpha_{1, i-1 / 2}^{R}\right)\right)
$$

Where $\alpha_{1, i+1 / 2}^{L}$ and $\alpha_{1, i-1 / 2}^{R}$ are obtained from the high order variable reconstruction.

For the convenience of coding, a modified form of the above expression is used and showed below:

$$
\alpha_{1, i}^{n+1}-\alpha_{1, i}^{n}=-\frac{\Delta t}{\Delta x}\left(A^{+} \Delta \alpha_{1, i-1 / 2}+A\left(\alpha_{1, i}-\alpha_{1, i-1 / 2}^{R}\right)+A^{-} \Delta \alpha_{1, i+1 / 2}+A\left(\alpha_{1, i+1 / 2}^{L}-\alpha_{1, i}\right)\right)
$$

When the Riemann problem has been solved at the position i-1/2, term $A^{+} \Delta \alpha_{1, i-1 / 2}$ and $A\left(\alpha_{1, i}-\alpha_{1, i-1 / 2}^{R}\right)$ can be obtained.

\subsection{Positivity preserving}

Severe robustness problems were encountered when solving two-phase problems in high speed conditions. When high-speed air flows around a liquid droplet or jet, there will be low-pressure areas on the leeward side of the liquid as illustrated in the Figure 1. And negative pressure always appears in these areas near the interface because of the large jump of the states across the material interface. The simulation can continue running with negative pressure in virtue of the $p_{\infty}$ which comes from the stiffened EOS $\left(p=\rho \varepsilon(\gamma-1)-\gamma p_{\infty}\right)$. However, problem appears in the high order variable reconstruction near these areas. The pressure reconstructed from the high order method cannot guarantee the positivity of the term $p+p_{\infty}$ which is needed in the calculation

of the sonic speed $\left(c=\sqrt{\gamma\left(p+p_{\infty}\right) / \rho}\right)$. Therefore, a positivity preserving process is required, and a MOON-type positivity preserving method $[42,43]$ is adopted. In the 
reconstruction process, the reconstruction method will be switched to a lower order method when negative density or $p+p_{\infty}$ is detected. The positivity preserving enhances the robustness of the simulation a lot.

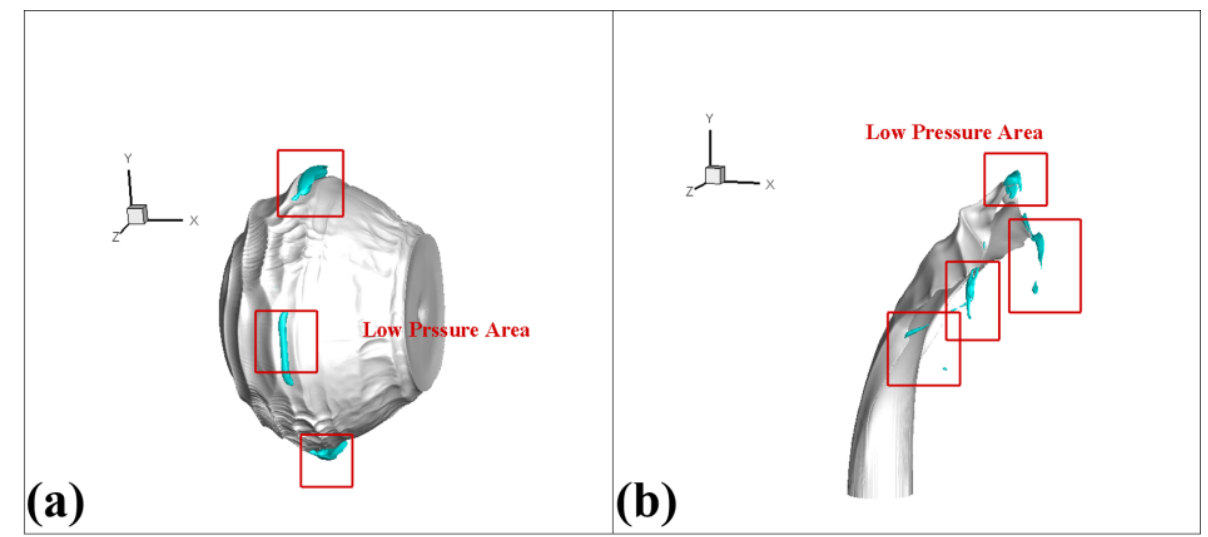

Figure 1 Low pressure areas represented by the blue iso-surface in simulations of two-phase flows; a) liquid droplet breakup in a supersonic flow; b) liquid jet in a supersonic crossflow; white iso-surface represents the liquid

\subsection{Adaptive mesh refinement}

Contrary to the sharp interface method the five-equation model is considered as a diffused interface method which means that the interfaces in five-equation model cover several cells. Thus, it requires high resolution at the interfacial areas so that the precise interface movements can be captured. However even non-uniform fixed mesh cannot provide the solution to this problem because interfaces distribute dynamically and unevenly in the computational domains during the evolution of the two-phase flows. The AMR (Adaptive Mesh Refinement) algorithm is tailored to this requirement in the two-phase flow simulation. The simulation code applied in this paper was developed in the framework of an open source software, AMROC (Adaptive Mesh Refinement 
Object-oriented $\mathrm{C}++$ )[30]. The AMROC implements the structured AMR algorithm[29] with a parallelization strategy[31] based on hierarchical grid patches. The AMR hierarchy consists of a sequence of levels $(i=0,1,2, \ldots)$ and refined grids are constructed from coarser ones based on fixed refinement ratios for each level. Though the refinement ratios are supposed to be constant along the time, they can vary among different levels as Figure 2 shows. With refinement ratios, we have $\Delta t_{i}=\Delta t_{i-1} / r_{i}$ which is stable for all levels. A recursive algorithm is adopted because the coarser level i-1 values at discrete times $t$ and $t+\Delta t_{0}$ are needed in the boundary updating on finer level i during $r_{i}$ time steps. In the recursive process, the fluxes on the coarse level are replaced by the fine level fluxes to ensure the conservation. And the reformed advection equation mentioned in the section 3.1 is not suitable for the conservative flux correction. Therefore, the wave propagation method was applied to solve the advection equation as stated above.

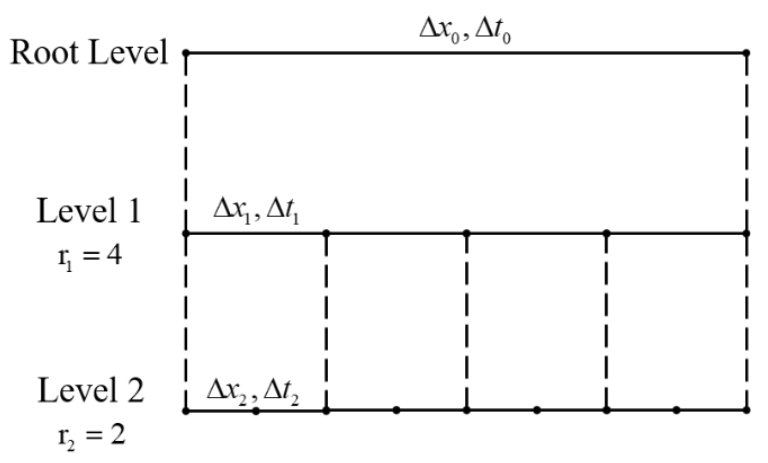

Figure 2 AMR hierarchy

\subsection{Two-phase simulation test}

Although the numerical methods applied in this paper have already been validated in the previous work[32], the tests in the current section is still required to check the 
validation of the AMR framework and enhancement of the efficiency. The conditions presented in the Table 2 were same with experiments by Sembian et al.[44] in which a water column was hit by an incident shock wave. In all three tests, the interface between water column and gas was resolved with 500 cells per diameter within a $[-2,2] \times[-2,2]$ zone where the length is nondimensionalized with water column diameter. No mesh refinement was applied in the test 3 while two and one levels of refinement were taken in the test 1 and 2 respectively. And magnitude of density gradient was taken as the refinement criterion.

Table 2 test setup

\begin{tabular}{cccc}
\hline & \multicolumn{2}{c}{ Gas } & Liquid droplet \\
\hline & After shock & Before shock & \\
Density $\left(\mathrm{kg} / \mathrm{m}^{3}\right)$ & 3.85 & 1.2 & 1000.0 \\
Pressure $(\mathrm{kPa})$ & 664.0 & 101.0 & 101.0 \\
Velocity $(\mathrm{m} / \mathrm{s})$ & 567.3 & 0 & 0 \\
\hline \multicolumn{4}{c}{ AMROC parameters } \\
\hline Total Levels & Test 1 & Test 2 & Test 3 \\
Refinement ratio $\mathrm{r}_{1}$ & 3 & 2 & 1 \\
Refinement ratio $\mathrm{r}_{2}$ & 2 & 4 & - \\
x-direction (cells) & 2 & - & - \\
y-direction (cells) & 250 & 250 & 2000 \\
\hline
\end{tabular}

The numerical schlieren images in Figure 3 compare the detailed structures captured in three tests, and Figure 4 illustrates the calculation time of three tests under parallel computations with 120 CPUs. The reflected shock wave in the upstream, the shock wave passing the water column and the expansion wave inside the water column are main phenomena in the test problem. As Figure 3 demonstrates, all three tests reach the same grid resolution at the highest level of the gird. And the details of the three main structures mentioned above are captured in all three tests which proves that the AMR 
strategy is an effective way to replace the fixed grid distribution with the same space resolution. In addition, the Figure 4 shows that the tests in which the AMR was applied required much less calculation time under certain computational resources. And the comparison between test 1 and test 2 illustrates that setting more levels to reach the same space resolution results in less integration time. However, the test with more levels required more time to accomplish the AMR procedures like generation and degeneration of grids. Therefore, proper level number and refinement ratios are requested to obtain the greatest enhancement of the efficiency. In the later sections, the selection of the level number and refinement ratios was based on the balance between the demand of space resolution and utilization efficiency of computational resources.

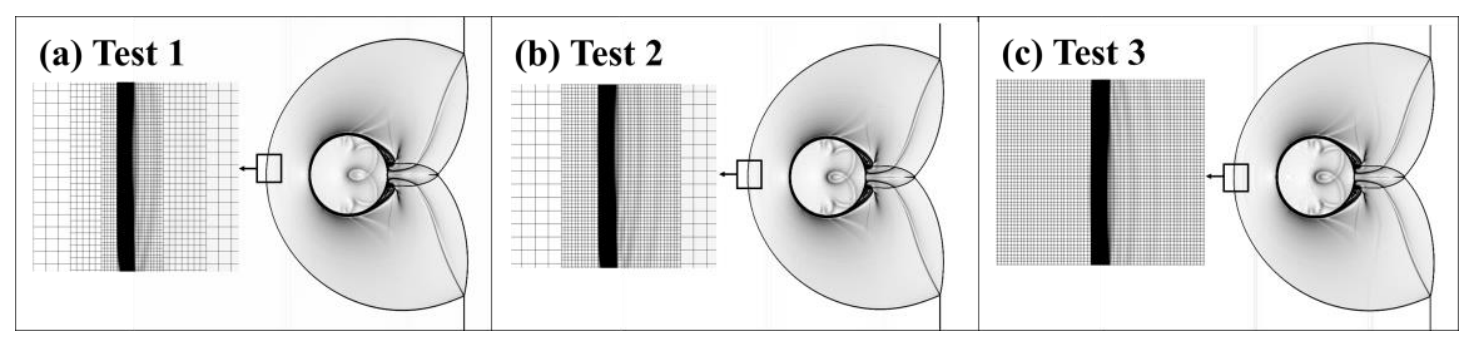

Figure 3 numerical schlieren contours of the results in three tests

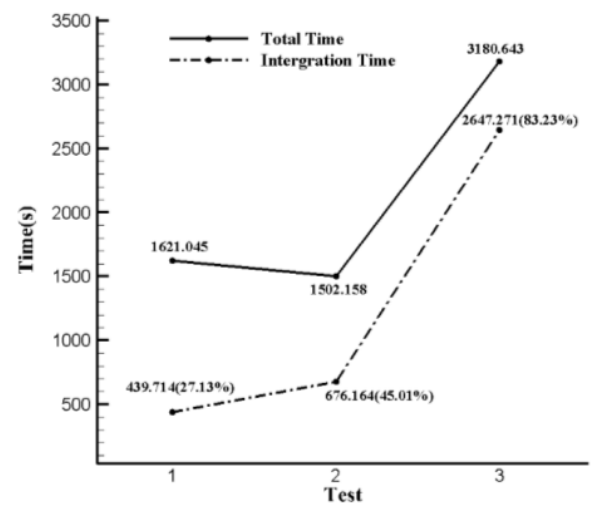

Figure 4 Efficiency comparison among three tests 


\section{Results}

\subsection{Simulation Settings}

Pure and periodically injected liquid jets in a supersonic crossflow were simulated to study the primary breakup process of the liquid jet in different injection conditions. A Ma 1.5 supersonic crossflow was taken as the standard case whose condition was listed in the Table 3. In the computational domain, the grid was refined with two levels at the liquid-gas interface where the resolution reached 160 cells per injector nozzle diameter. And the center of injector nozzle was placed at the position $(\mathrm{x}, \mathrm{y}, \mathrm{z})=(0,0,0)$ with liquid injected in the y-direction and supersonic gas flowing in the x-direction. The grid distribution varies during the process of computations under the AMR strategy. The instantaneous grid distribution is illustrated in Figure 5 where grid is refined at the interfaces represented by the red density isolines.

Table 3 supersonic crossflow conditions and AMR settings

\begin{tabular}{ccccc}
\hline \multicolumn{5}{c}{ Supersonic crossflow } \\
\hline Mach number, $M_{\infty}$ & 1.5 & Velocity, $u_{\infty}(\mathrm{m} / \mathrm{s})$ & 406.3 \\
Static pressure, $P_{\infty}(\mathrm{kPa})$ & 52.4 & Gas density, $\rho_{\infty}\left(\mathrm{kg} / \mathrm{m}^{3}\right)$ & 1.0 \\
\hline \multicolumn{5}{c}{ AMR parameters } \\
\hline Total Levels & 3 & x-direction & $-2.2 \sim 4.3$ & 130 cells \\
Refinement ratio $\mathrm{r}_{1}$ & 4 & y-direction & $0.0 \sim 4.5$ & 90 cells \\
Refinement ratio $\mathrm{r}_{2}$ & 2 & z-direction & $-2.0 \sim 2.0$ & 80 cells \\
Length is nondimensionalized with injector nozzle diameter (d=1.0mm)
\end{tabular}




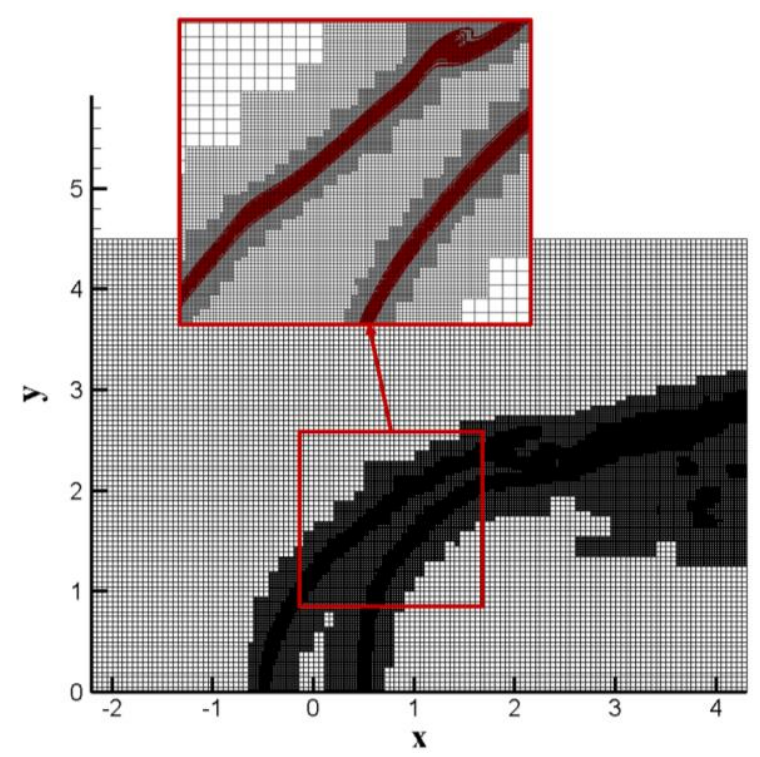

Figure 5 instantaneous grid distribution of the slice at $Z=0$ during the process of computation with AMR strategy

\subsection{Primary breakup in the near field}

A lot of work has been carried out to study the primary breakup of liquid jet in a low-speed crossflow. However, high-speed gas flows and high liquid-gas density ratio are still two challenges in the simulation of liquid jet in a supersonic crossflow. In experiments, the near-filed is always covered by the dense groups of small droplets striped from the liquid column. The optical images from experiments are incapable to capture the behavior of the liquid column. In this section, primary breakup of a pure liquid jet in a supersonic crossflow is studied based on the simulation results. And the conditions of pure liquid jet are given as Table 4 shows.

Table 4 Pure liquid jet conditions

\begin{tabular}{lccc}
\hline Injection velocity, $\mathrm{v}_{l}(\mathrm{~m} / \mathrm{s})$ & 15.9 & Water density, $\rho_{l}\left(\mathrm{~kg} / \mathrm{m}^{3}\right)$ & 1000.0 \\
& & Momentum ratio, $q=\rho_{l} v_{l}^{2} / \rho_{\infty} u_{\infty}^{2}$ & 1.53 \\
\hline
\end{tabular}


Evolution of a pure liquid jet in a Ma 1.5 supersonic crossflow is demonstrated in the Figure 6. As Figure 6 shows, obvious surficial waves were captured on the windward side of the liquid column in the simulation. As the top view shows, liquid column is stretched into a liquid film with the instable waves traveling on the liquid surface. The surficial waves travel in two directions as showed in Figure 7 where two kinds of vortex, v1 and v2, are generated along those two kinds of instabilities. As a result, the two kinds of instabilities are responsible for the primary breakup of the liquid jet in different modes. The two kinds of modes are named column breakup along the injection direction and surface breakup cross the injection direction. Although the two modes act in different directions, they interact with each other in the development of the breakup process. The instabilities causing surface breakup help liquid column stretching thinner, while the interaction between two direction instabilities results in the liquid striped from the sides of the liquid column swirling away downstream. The details of the column breakup and surface breakup are going to be discussed in the following sections. 


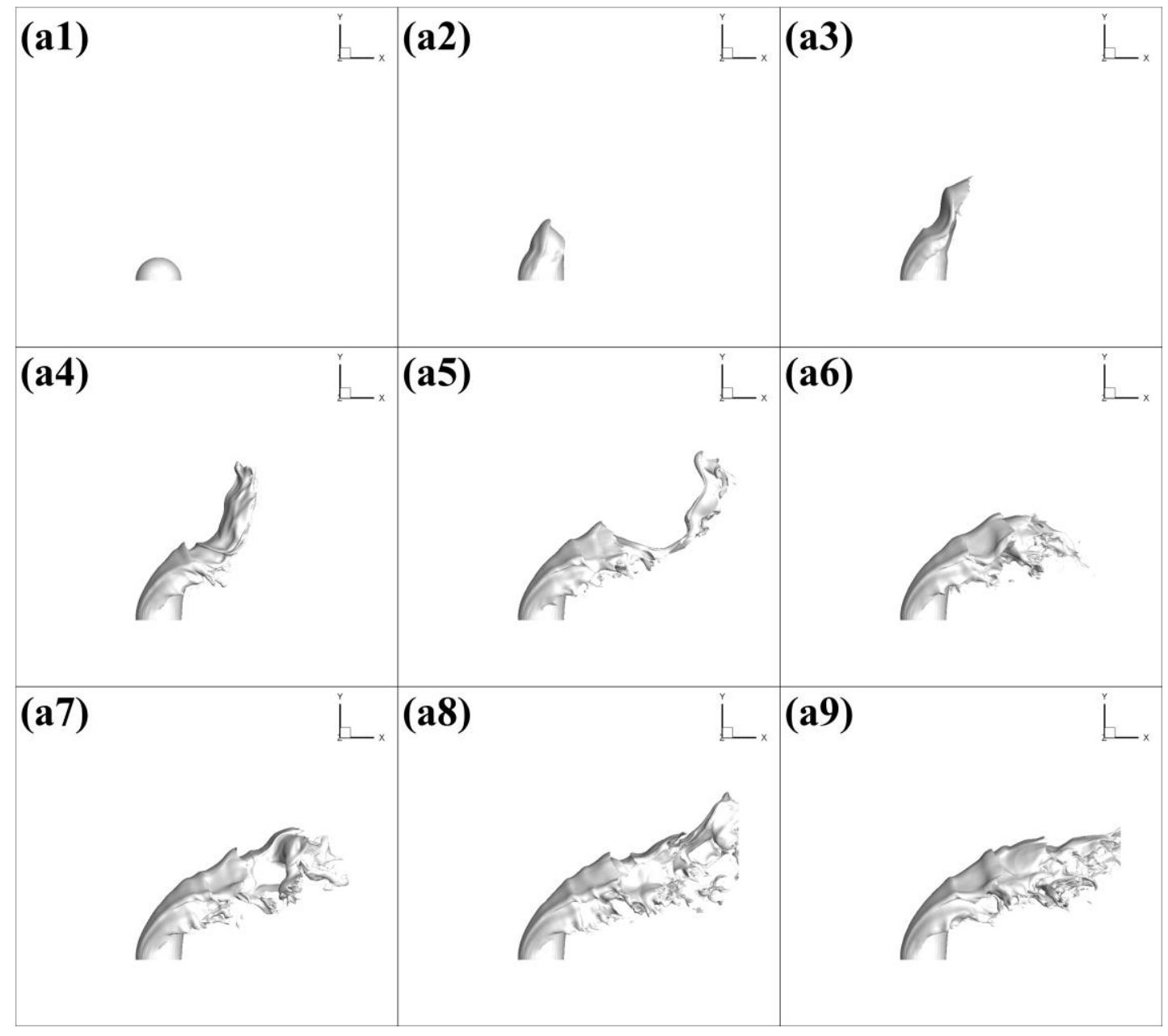




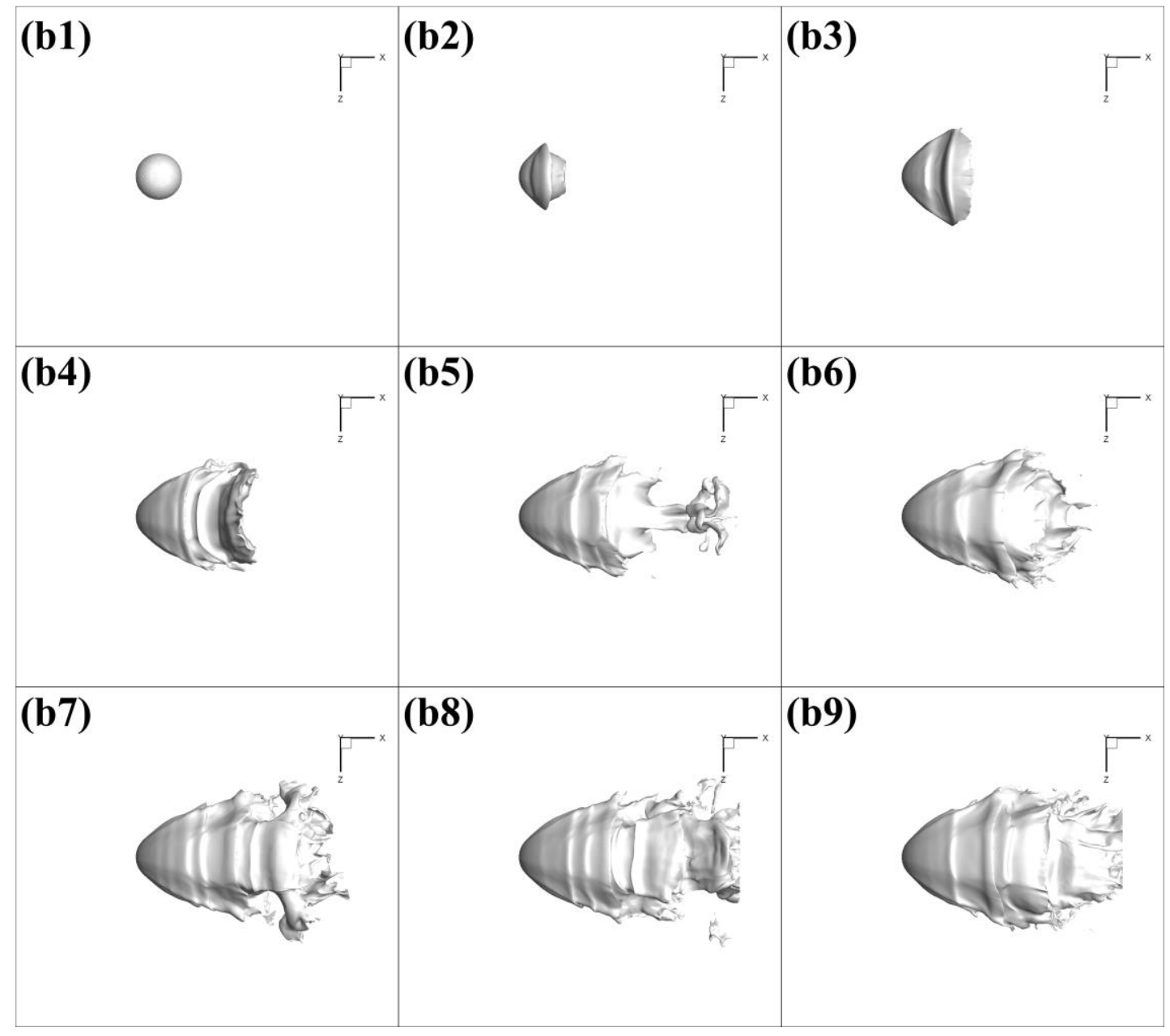

Figure 6 primary breakup process of a pure liquid jet in a supersonic crossflow, $\Delta \mathrm{t}=20 \mu \mathrm{s}$, (a)side view, (b)top view

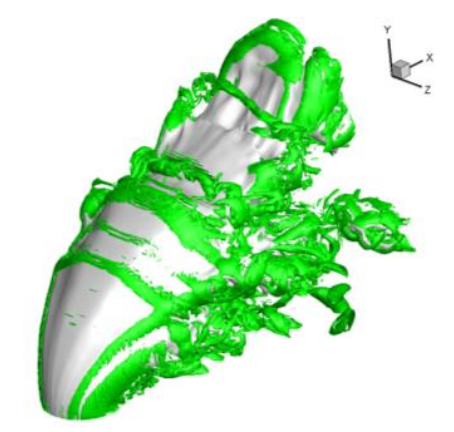

Figure 7 vortices displayed by $Q$ criterion

\subsubsection{Mode1-column breakup}

Though this mode is called column breakup, the liquid has already been stretched 
into a thin film actually and the spanwise development of the liquid film is also a result of transversely travelling waves which will be discussed later. At first, the development of the instabilities on the windward surface will be studied as Figure 8 demonstrates. The unstable waves appeared on the windward surface, shortly after the liquid was injected from the injector. And these instabilities traveled with the liquid moving up. At the height where the liquid was bent to be parallel with the crossflows, three vortices illustrated in the Figure 8(1) were generated behind the unstable wave identified by the solid or dashed square frames in the Figure 8. And the vortex in the middle was reverse to the other two vortices. In the developing, these vortices curled up the liquid surface which resulted in the stretching of the liquid and a wavier surface. In turn, the thinner and wavier liquid would enhance the turbulence of the gaseous flows. When the liquid film was too thin to hold in coherence under the strong aerodynamic interactions, the liquid would finally breakup. The pressure difference between the windward and leeward of the liquid bent over the liquid column and then the force bending the liquid would become one to prevent the liquid from moving higher in the Y-direction. Therefore, the Y-direction velocity of the main liquid flow reduced when liquid reached a higher position as Figure 9 shows. Moreover, the Figure 9 also illustrates that some parts of the wavy liquid were accelerated up or down from the main flow. As a result of the liquid flowing in opposite directions, the thin liquid film reached the column breakup point. 
(1)

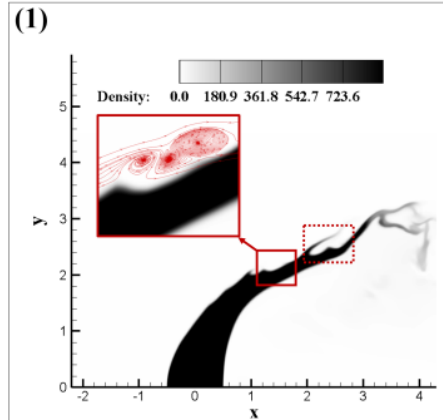

(3)

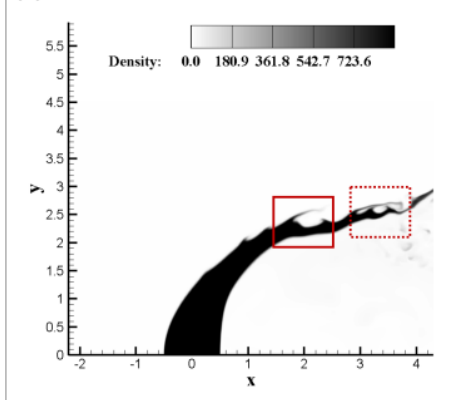

(2)

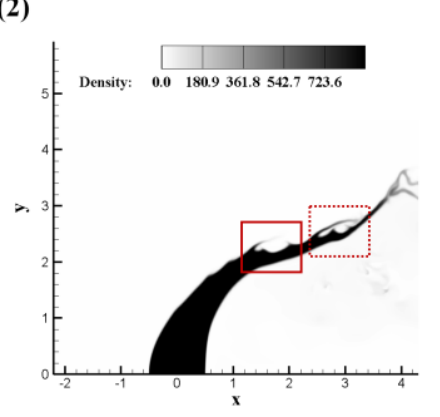

(4)

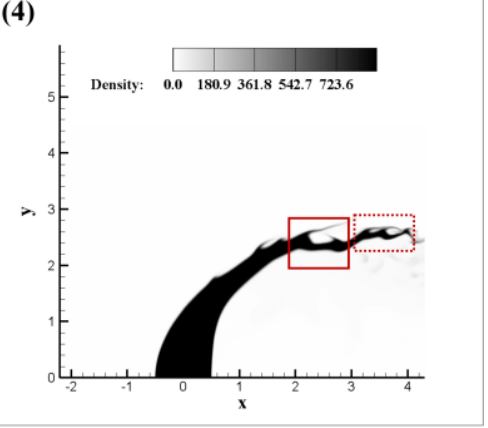

Figure 8 development of the instabilities along the injection direction,

$\operatorname{density}\left(\mathrm{kg} / \mathrm{m}^{3}\right)$ contours of slice at $\mathrm{Z}=\mathbf{0}, \Delta \mathrm{t}=\mathbf{0 . 2} \mu \mathrm{s}$

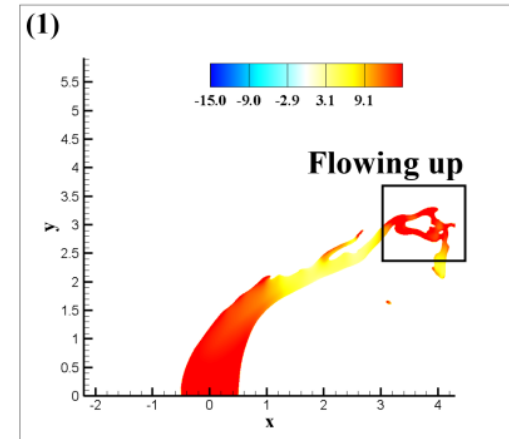

(2)

(3)

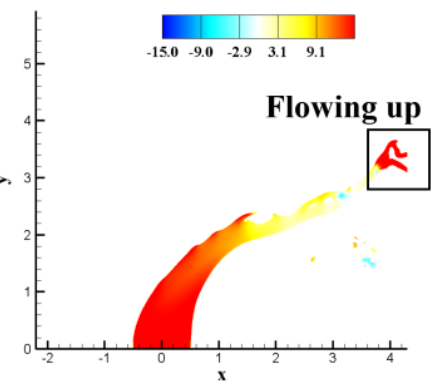

(4)
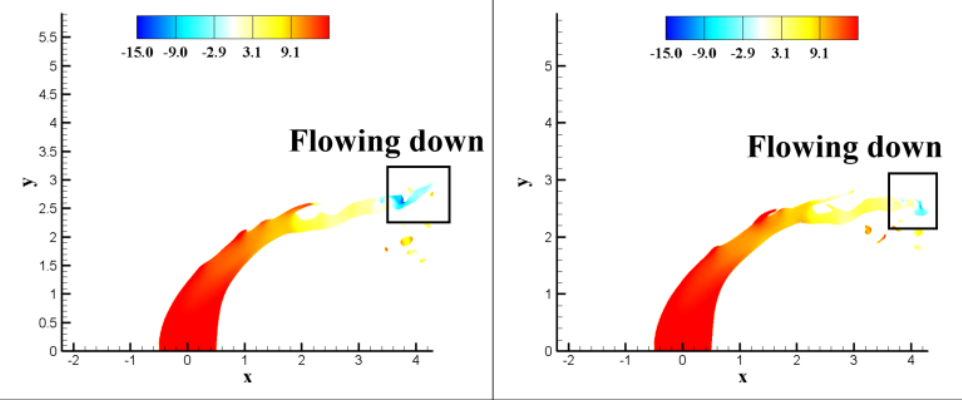

Figure 9 distribution of liquid velocity-in-Y-direction $(\mathrm{m} / \mathrm{s})$, slice at $\mathrm{Z}=\mathbf{0}, \Delta \mathrm{t}=\mathbf{2 0} \boldsymbol{\mu} \mathrm{s}$ 


\subsubsection{Mode2-surface breakup}

The waves traveling in the spanwise direction caused another breakup process in the near-field. Figure 10 demonstrates three slices taken from positions $Y=1 d, 1.5 \mathrm{~d}$ and $2 \mathrm{~d}$ where $\mathrm{d}$ represents the diameter of liquid injector as mentioned in section 4.1. As Figure 10 shows, liquid was stripped from the two sides of the liquid column which is similar to the behavior of the liquid drop breakup in a supersonic flow studied in our previous work[32]. The liquid stripped off the surface was whirled downstream by the vortices generated around the liquid column. The morphologies of the liquid column at different positions have their corresponding stages in liquid drop breakup. At the place near the injector nozzle, the instable waves appeared on the windward surface of the liquid and travel towards the two sides of the liquid column. Along with transportation of the waves, liquid gathered together and then was stripped off the column by highspeed gas flows. And at a higher position, the liquid column was flattened thinner by the pressure difference between windward and leeward sides. The liquid column finally developed into a liquid film which broke up under the action of the mode 1 . The scale of the surface breakup is much smaller than the space resolution of the $3 \mathrm{~d}$ simulation. The surface breakup is under the influence of the flows in streamwise direction and injection-direction. Ignoring the flows in the injection-direction can simplify the surface breakup into a $2 \mathrm{~d}$ problem. And in consideration of the similarity between surface breakup process and the liquid drop breakup in a supersonic flow, a $2 \mathrm{~d}$ simulation with high space resolution (1000 cells per diameter length at interfaces) was carried out to study the cross-section deformation of the liquid column and the 
conditions are the same with the standard case which has been presented above while AMR settings are given in the Table 5. The $2 \mathrm{~d}$ simulation is a simplification of the $3 \mathrm{~d}$ physical problem and only the influence of the streamwise flows is taken into account. The $2 \mathrm{~d}$ simulation can only interpret parts of the factors that cause the surface breakup. A completed mechanism of the surface breakup still needs a further study based on a more accurate $3 \mathrm{~d}$ simulation. With the high space resolution, the small size instabilities on the windward surface were captured as Figure 11 shows. As a symbol of the supersonic crossflow, a shock was appeared in front of the liquid. After shock, the gaseous flows varied from subsonic flows on the windward side to supersonic flows on the leeward side. And the initial instabilities gathered in the transonic zone as Figure 11(b) demonstrates. Following the [45], we apply the small-perturbation theory to do the analysis. In the case of the compressible flow over an interface defined by $y=\kappa \sin (\alpha x)$ in which the $\varepsilon$ is extremely small, the pressure coefficient can be obtained by the following equations[45]:

$$
\frac{p-p_{\infty}}{\frac{1}{2} \rho u_{\infty}^{2}}=-\frac{2 \kappa \alpha \sin (\alpha x)}{\sqrt{1-M a^{2}}}, M a<1 ; \frac{p-p_{\infty}}{\frac{1}{2} \rho u_{\infty}^{2}}=-\frac{2 \kappa \alpha \cos (\alpha x)}{\sqrt{M a^{2}-1}}, M a>1
$$

The pressure varies along the wavy interface. And the amplitude of the pressure variation represents the influence of the instability. From the equation (18), we can have that the amplitude of the pressure variation is in-phase with the instability in subsonic flow while out-of-phase with the instability in supersonic flow. Therefore, the pressure variation caused by instability is enhancing the instability in return in the subsonic flow and suppressing the instability in the supersonic flow. This theory explains why the 
initial instabilities gathering in the transonic zone. In addition, the transonic flow itself exhibit an oscillatory character[46], thus the liquid exposed in the transonic flow encounters stronger unstable actions. A comparison between $3 \mathrm{~d}$ and $2 \mathrm{~d}$ simulations are illustrated in the Figure 12. The image in the Figure 12(b) was taken at the $30 \mu$ s and it is considered to be approximately corresponding with the liquid cross-section at the position of $0.5 \mathrm{~d}\left(30 \mu s \times v_{l}\right)$. Although the space resolution of the $3 \mathrm{~d}$ simulation was insufficient to capture the small size instabilities on the liquid, three characteristic structures was obtained as marked in the Figure 12. Near the stagnation point, the liquid remained smooth and no surface breakup happened in this region. The liquid was stripped off the main liquid column at the liquid edge where the large-size surface breakup which can even be captured in the $3 \mathrm{~d}$ simulation occurred. And a stage structure connected above two parts. The stage structure was formed under the action of the transonic flow. Within the stage structure, small-size surface breakup happened. The $3 \mathrm{~d}$ simulation was not able to capture these small-size processes because of the insufficient space resolution. 


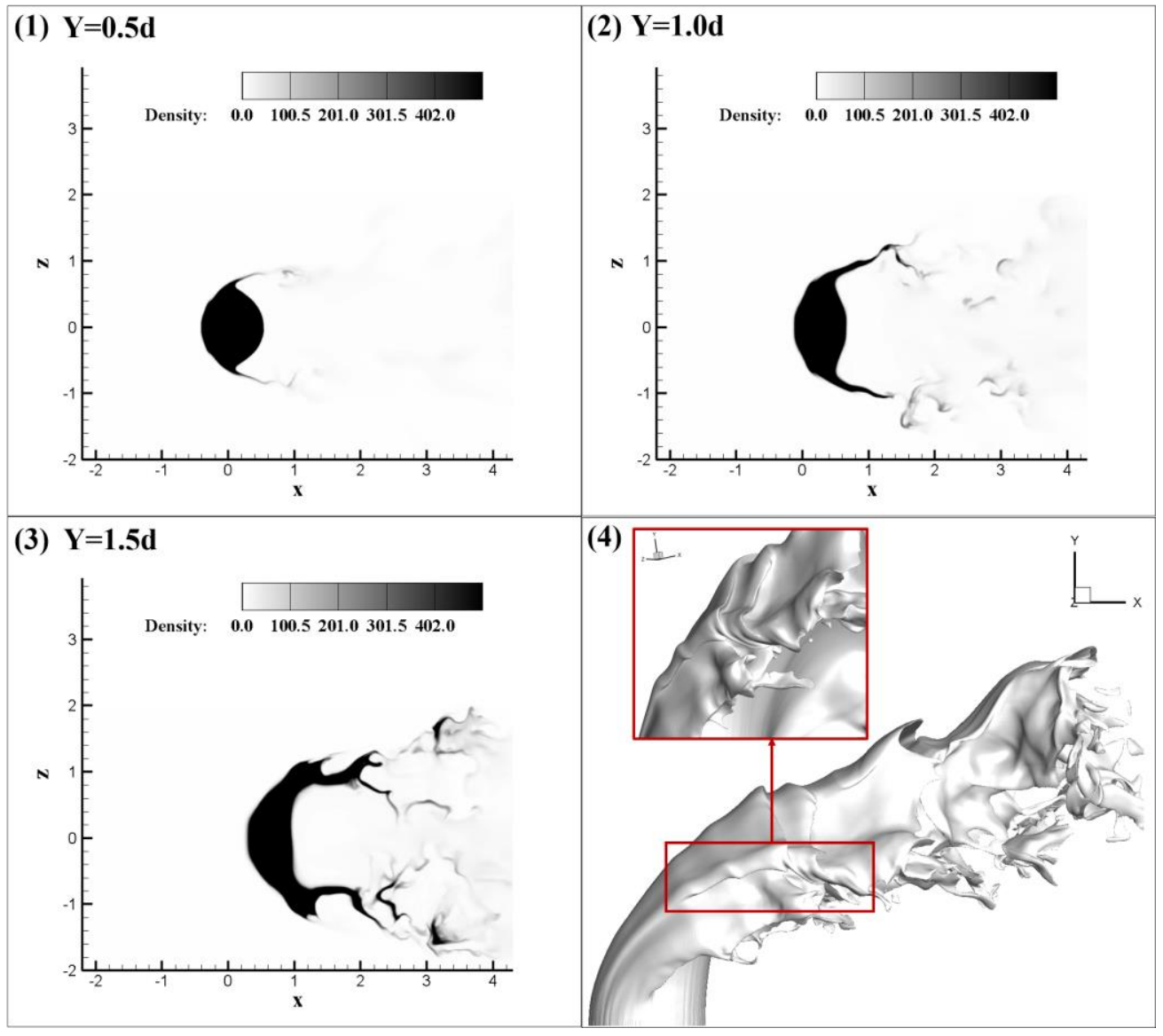

Figure 10 surface breakup due to transverse waves at $t=260 \mu s ;$ density

$\left(\mathrm{kg} / \mathrm{m}^{3}\right)$ contours of slices at different positions

Table 5 AMR settings of $2 d$ simulations

\begin{tabular}{cc|ccc}
\hline \multicolumn{5}{c}{ AMR parameters } \\
\hline Total Levels & 2 & x-direction & $-2.0 \sim 2.0$ & 1000 cells \\
Refinement ratio $\mathrm{r}_{1}$ & 4 & z-direction & $-2.0 \sim 2.0$ & 1000 cells \\
Length is nondimensionalized with injector nozzle diameter $(\mathrm{d}=1.0 \mathrm{~mm})$ \\
\hline
\end{tabular}


(a)

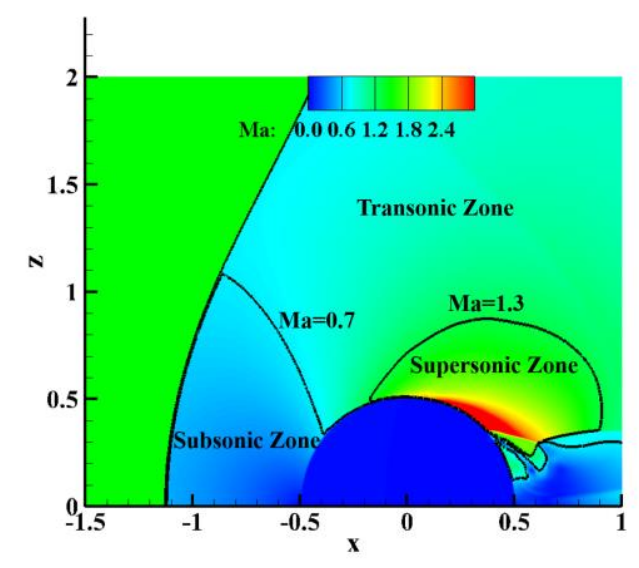

(b)

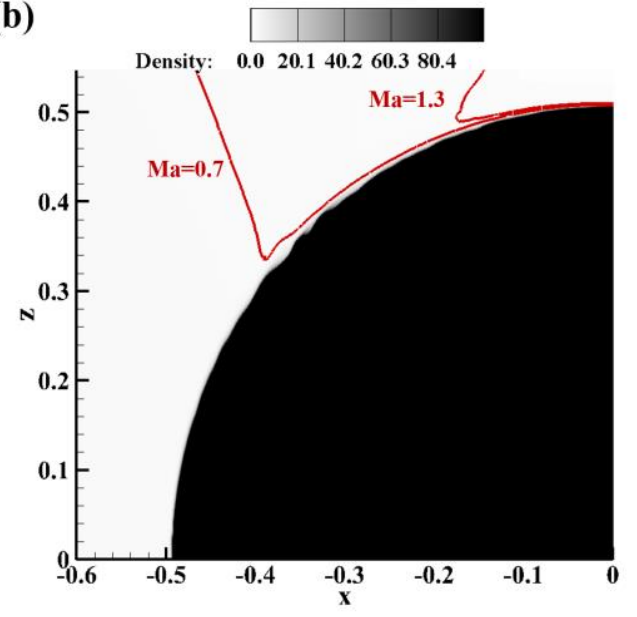

Figure 11 Mach number distribution in 2d simulation, (a)Mach number contours, (b)density $\left(\mathrm{kg} / \mathrm{m}^{3}\right)$ contours

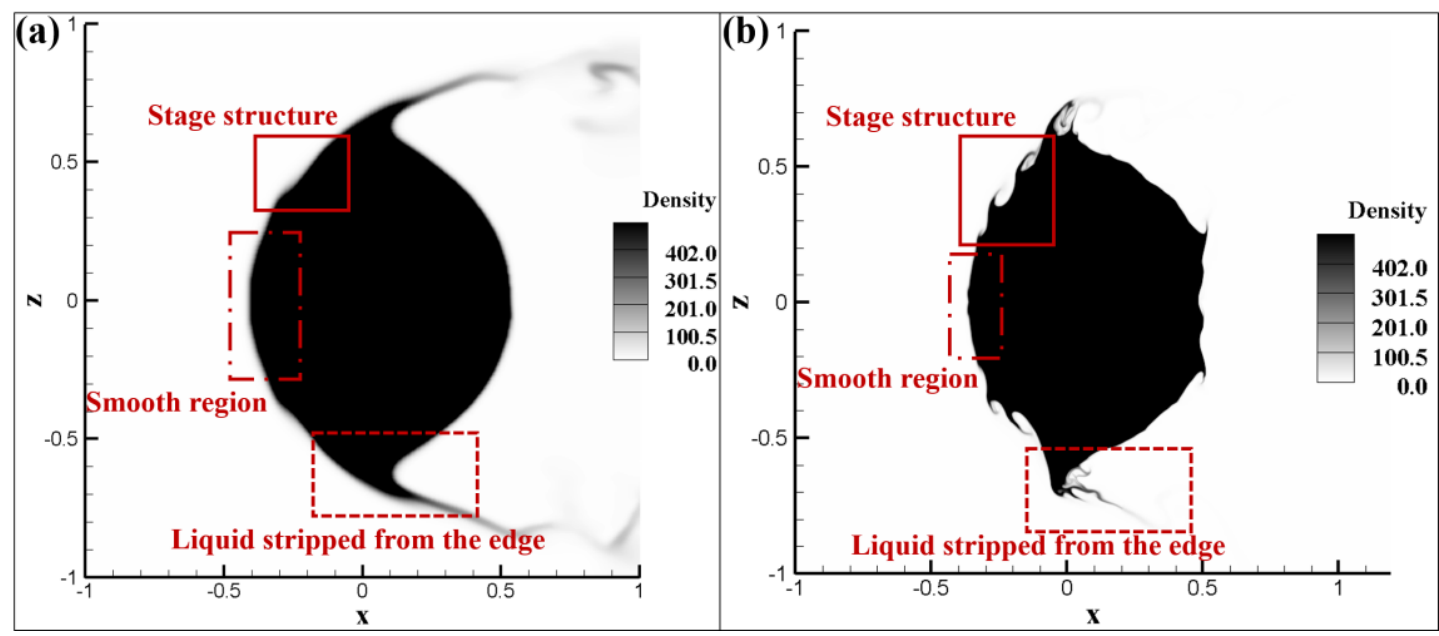

Figure 12 comparison between $3 \mathrm{~d}(\mathrm{a})$ and $2 \mathrm{~d}(\mathrm{~b})$ simulations, $\operatorname{density}\left(\mathrm{kg} / \mathrm{m}^{3}\right)$

contours

\subsection{Effect of the Mach number}

Table 6 Ma 1.8 supersonic crossflow conditions

\begin{tabular}{lccc}
\hline \multicolumn{4}{c}{ Supersonic crossflow } \\
\hline Mach number, $M_{\infty}$ & 1.8 & Velocity, $u_{\infty}(\mathrm{m} / \mathrm{s})$ & 487.5
\end{tabular}




\begin{tabular}{lccc} 
Static pressure, $P_{\infty}(\mathrm{kPa})$ & 52.4 & Gas density, $\rho_{\infty}\left(\mathrm{kg} / \mathrm{m}^{3}\right)$ & 1.0 \\
\hline & Liquid jet flow \\
\hline Injection velocity, $\mathrm{v}_{l}(\mathrm{~m} / \mathrm{s})$ & $19.1 \quad$ Water density, $\rho_{l}\left(\mathrm{~kg} / \mathrm{m}^{3}\right)$ & 1000.0 \\
Water pressure, $p_{l}(\mathrm{kPa})$ & 827.0 & Momentum ratio, $q=\rho_{l} v_{l}^{2} / \rho_{\infty} u_{\infty}^{2}$ & 1.53 \\
\hline
\end{tabular}

A Ma 1.8 supersonic crossflow was introduced in this section to study the effect of gaseous flow Mach number while the momentum ratio was remained the same with the standard case as Table 6 demonstrates. In the condition of the supersonic crossflows, the Mach number represents not only the dynamic pressure but also the strength of the shock before the liquid jet which is a symbol of the liquid jet in a supersonic crossflow. Therefore, it is an important parameter of the fluid to have an obvious influence on the primary breakup of the liquid jet in a supersonic crossflow. In this section, a $2 \mathrm{~d}$ simulation was also carried out to study the cross-section deformation in the same way we did in the section 4.2.2. In consideration of different crossflow velocities, the discussion was based on the characteristic time T nondimensionalized by Equation (19). The same characteristic time roughly indicates that two cases of different conditions reach the same stage during the development of the flow fields when the two cases are at different physical time.

$$
T=t / t^{*}, t^{*}=\frac{d}{u_{\infty}} \sqrt{\frac{\rho_{l}}{\rho_{\infty}}}
$$

The Figure 13-14 present the 3d comparison between the Ma 1.5 case and the Ma 1.8 case in the side view and top view respectively. The comparison was carried out at the same characteristic time defined by Equation (19), because it is obvious that the Ma 1.8 breakup process was ahead of the Ma 1.5 in physical time. From the results of the 
$3 \mathrm{~d}$ simulations, larger size instabilities were captured in the Ma 1.8 case comparing to the Ma 1.5 case. In the Figure 15, slices taken from the position of $Z=0$ demonstrated the clear images of the instabilities along the injection direction. The momentum ratios are the same in the two cases, therefore there is no obvious difference on the penetration abilities of the liquid jet. However, it is apparent that the instability in the Ma 1.8 case possess a larger size. And the liquid film of the Ma 1.8 case was thinner than of the Ma 1.5 case at the same position above $\mathrm{Y}=1.5 \mathrm{~d}$. In addition, the Figure 16 shows the $\mathrm{Y}$ direction velocity difference with the injection velocity $(15.9 \mathrm{~m} / \mathrm{s}$ in case Ma 1.5 and $19.1 \mathrm{~m} / \mathrm{s}$ in case $\mathrm{Ma} 1.8$ ) at $\mathrm{T}=3.08$. With larger size instability, the unstable structure in the Ma 1.8 case obtained a greater velocity difference with the main liquid flow than in the Ma 1.5 case. As a result, the thinner liquid film and greater velocity difference with main flow lead to an easier column breakup in the Ma 1.8 case. The comparisons between cases Ma 1.5 and Ma 1.8 reveal that the Ma 1.8 crossflow provided a stronger aerodynamic force which resulted in a greater augmentation of the surficial instability. Besides, the comparisons were carried out based on the same characteristic time while the process in case Ma 1.8 happened much faster than in case Ma 1.5. This indicates that the higher Ma number crossflow possesses not only better acceleration ability but also greater augmentation of the instability. Cross-section structures at different positions are compared in the Figure 17. Similar with the column breakup process, the Ma 1.8 case obtained a more complicate and finer liquid flow field because the Ma 1.8 gaseous flow was greater in turbulence and aerodynamic actions. Following the section 4.2.2, a $2 \mathrm{~d}$ simulation was applied in the case of that the surface breakup size is much 
smaller than the column breakup size which means capturing surface breakup requires higher space resolution. In the Figure 18, the initial flow filed is compared. Though the Ma 1.8 case obtained a wider region of the transonic and the supersonic, the Ma number distributions of two cases near the liquid surface show no differences. Therefore, the initial instabilities of the two cases distribute in the same range as the Figure 18(a2) and 18(b2) illustrates. This situation in return proves the conclusion drew in the section 4.2.2. In the further development, the instabilities on liquid surface in the Ma 1.8 flow are finer in size and more in number because the Ma 1.8 incoming flow provided stronger turbulence and aerodynamic forces. To evaluate the breakup process in a quantitative way, the areas of liquid on the slice of $\mathrm{Y}-\mathrm{Z}$ plane along $\mathrm{X}$-direction are collected in the Figure 20 in which the results from $\mathrm{T}=2.80$ to $\mathrm{T}=3.65$ were averaged. When $\alpha>0$, the cell was taken into account of the liquid area. And the liquid was considered to be under breakup in the cell where $0<\alpha<0.5$. As Figure 20 shows, the liquid in case Ma1.8 was undergoing a greater breakup process and distributed in a wider range before $X=2$ around. In further downstream, distribution of liquid in the case Ma 1.5 expanded faster than in the case Ma 1.8. This is because that the crossflow of higher speed possessed a better ability of carrying the liquid downstream. Otherwise, the Ma 1.8 case reached a better atomization in the near-field as the lines before $\mathrm{X}=1.8$ illustrate. There are two peaks on the area lines of both Ma 1.5 case and Ma 1.8. And these inflection points are corresponding to the large-scale instabilities which are responsible for the breakup. The result indicates that the large-scale instabilities of the higher speed case were closer to the injection nozzle. 


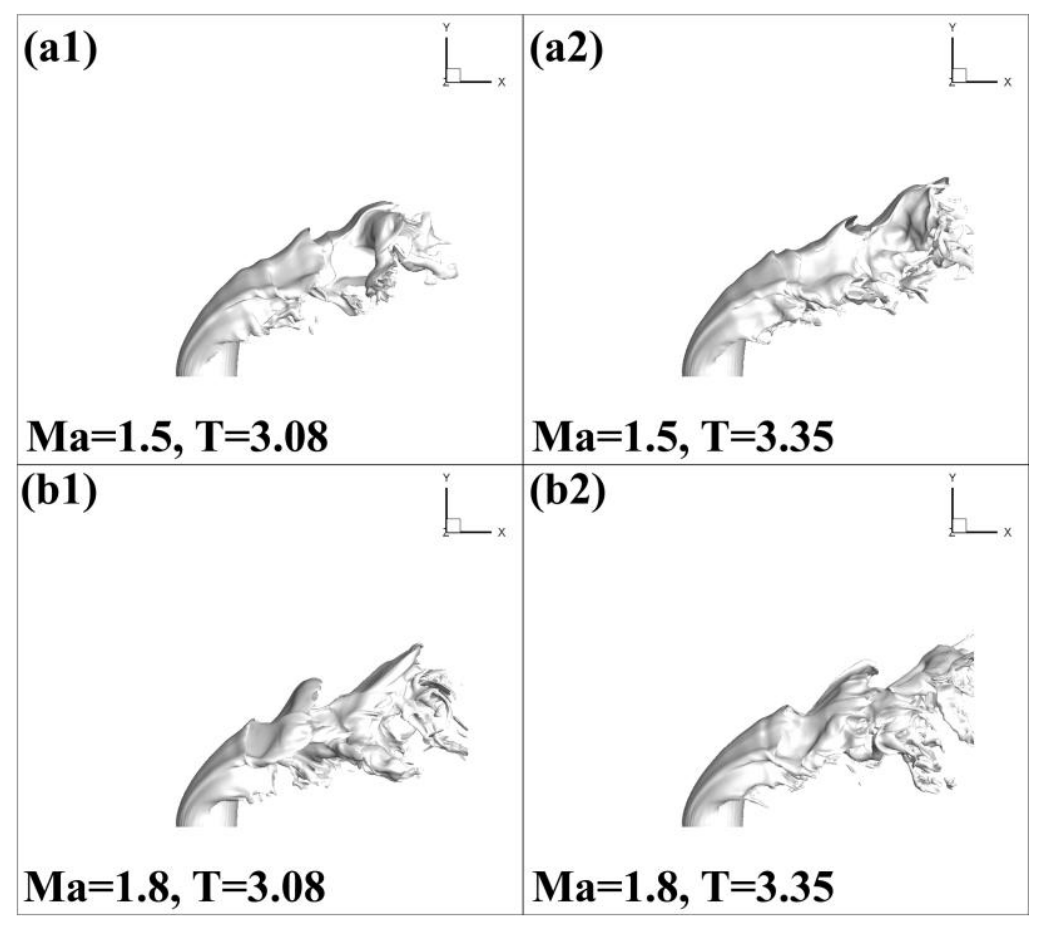

Figure 13 morphology comparison (side view) between cases of Ma 1.5 and 1.8

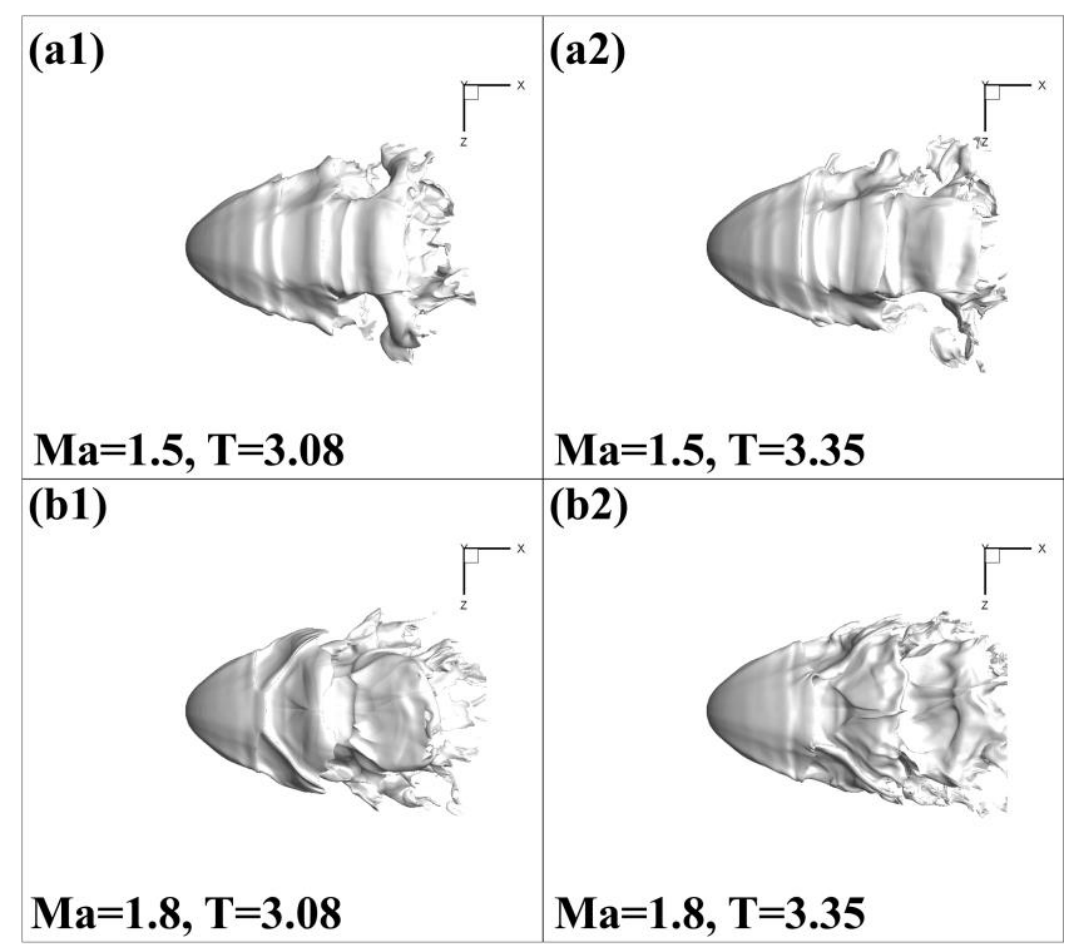

Figure 14 morphology comparison (top view) between cases of Ma 1.5 and 


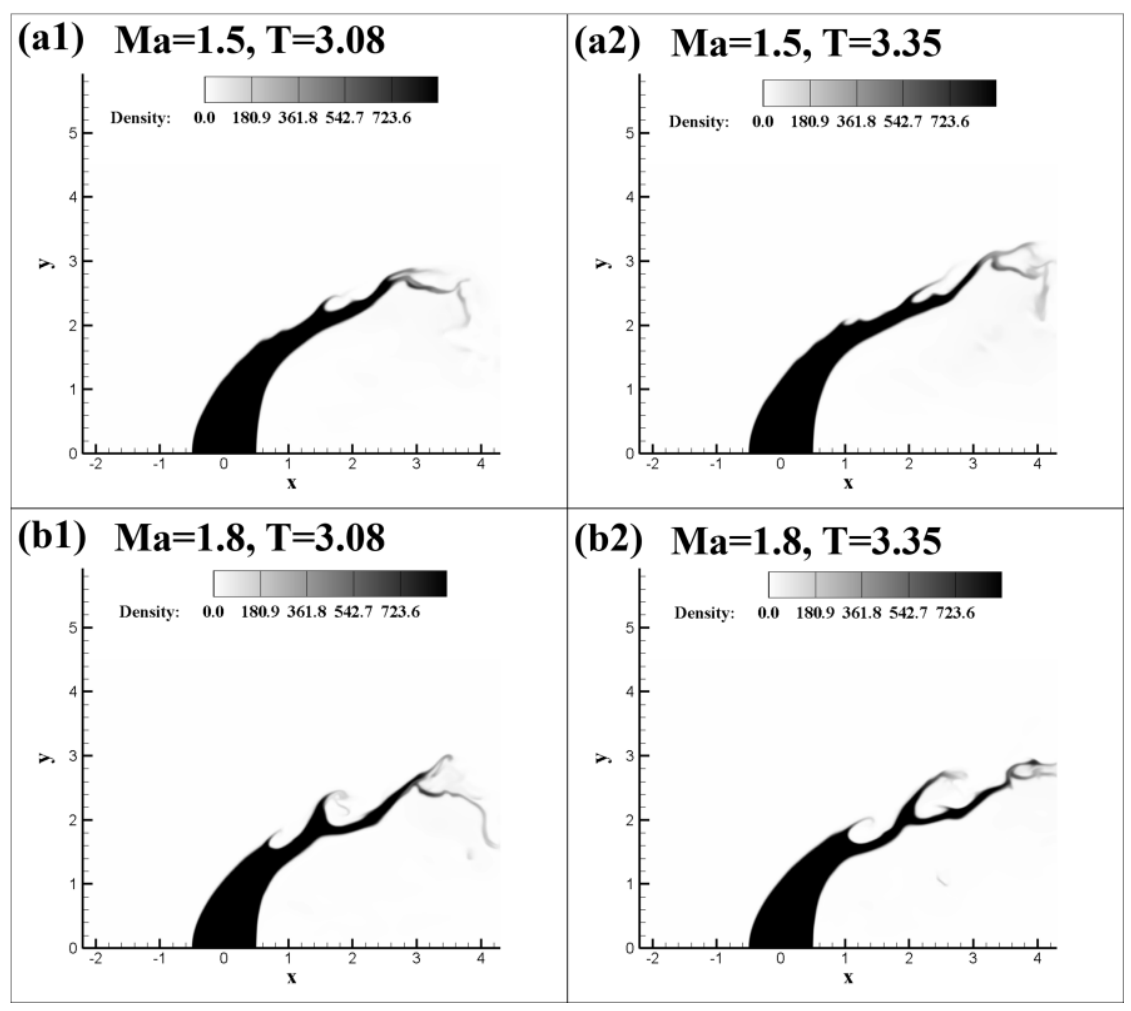

Figure 15 instabilities along injection direction at slice of $Z=0$, $\operatorname{density}\left(\mathrm{kg} / \mathrm{m}^{3}\right)$ contours

(a)

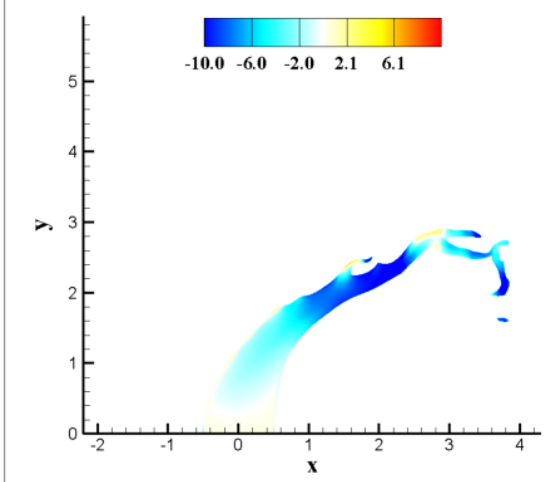

(b)

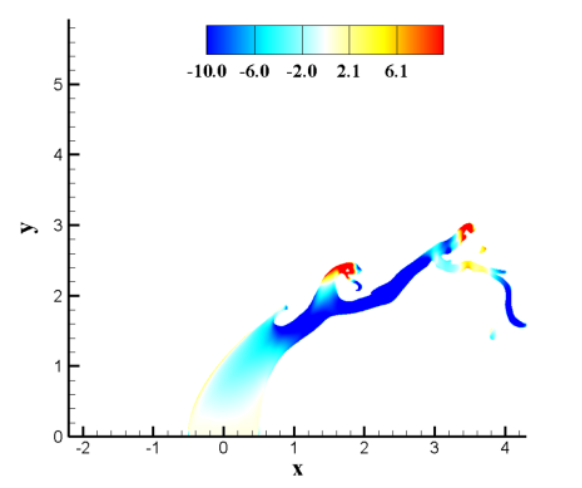

Figure 16 Y-direction velocity $(\mathrm{m} / \mathrm{s})$ difference with injection velocity at slice of $\mathrm{Z}=\mathbf{0}$ at $\mathrm{T}=3.08$, (a)Ma 1.5 and (b)Ma 1.8 


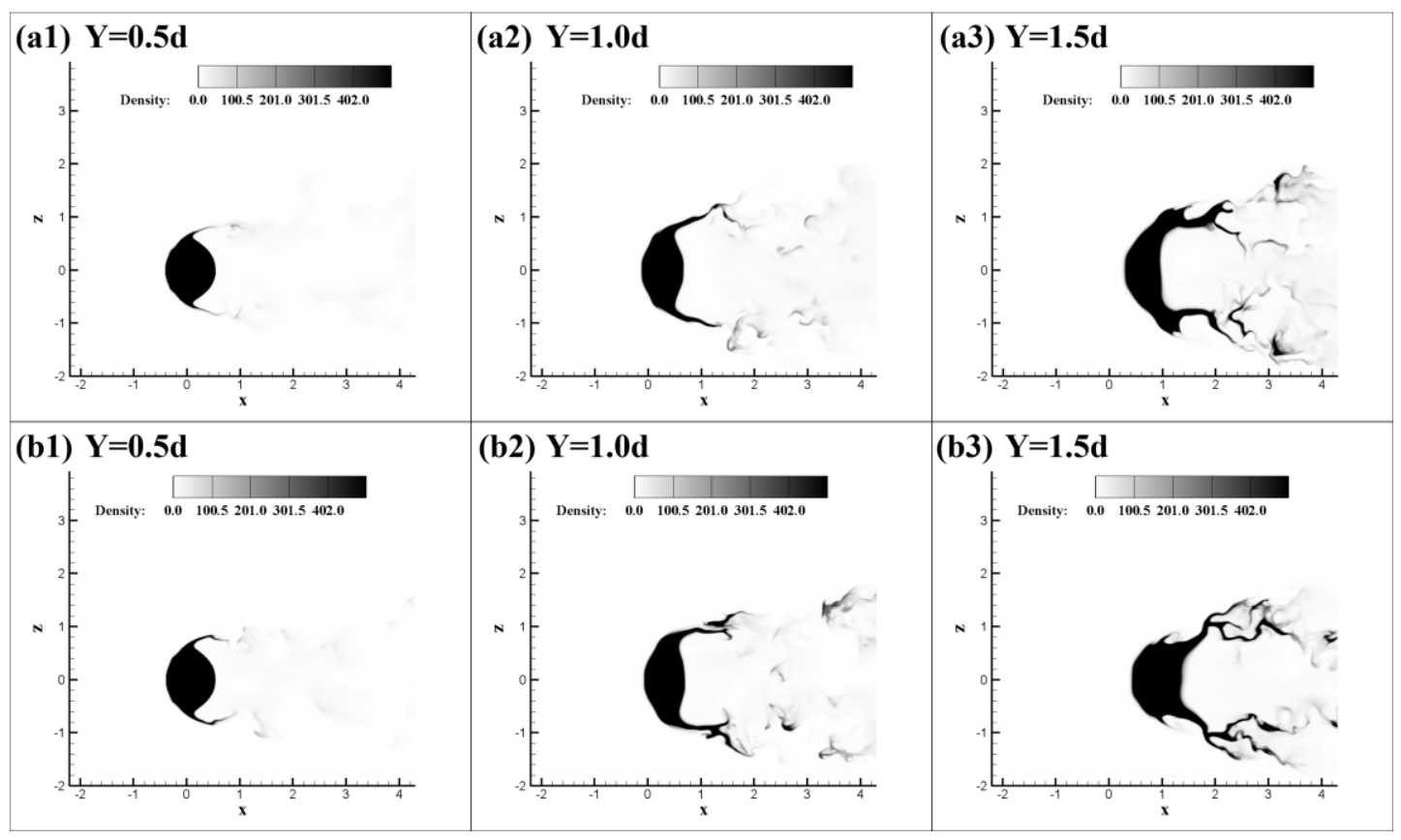

Figure 17 comparison of surface breakup process between case (a)Ma 1.5

and (b)Ma 1.8 at $\mathrm{T}=3.35$, density $\left(\mathrm{kg} / \mathrm{m}^{3}\right)$ contours

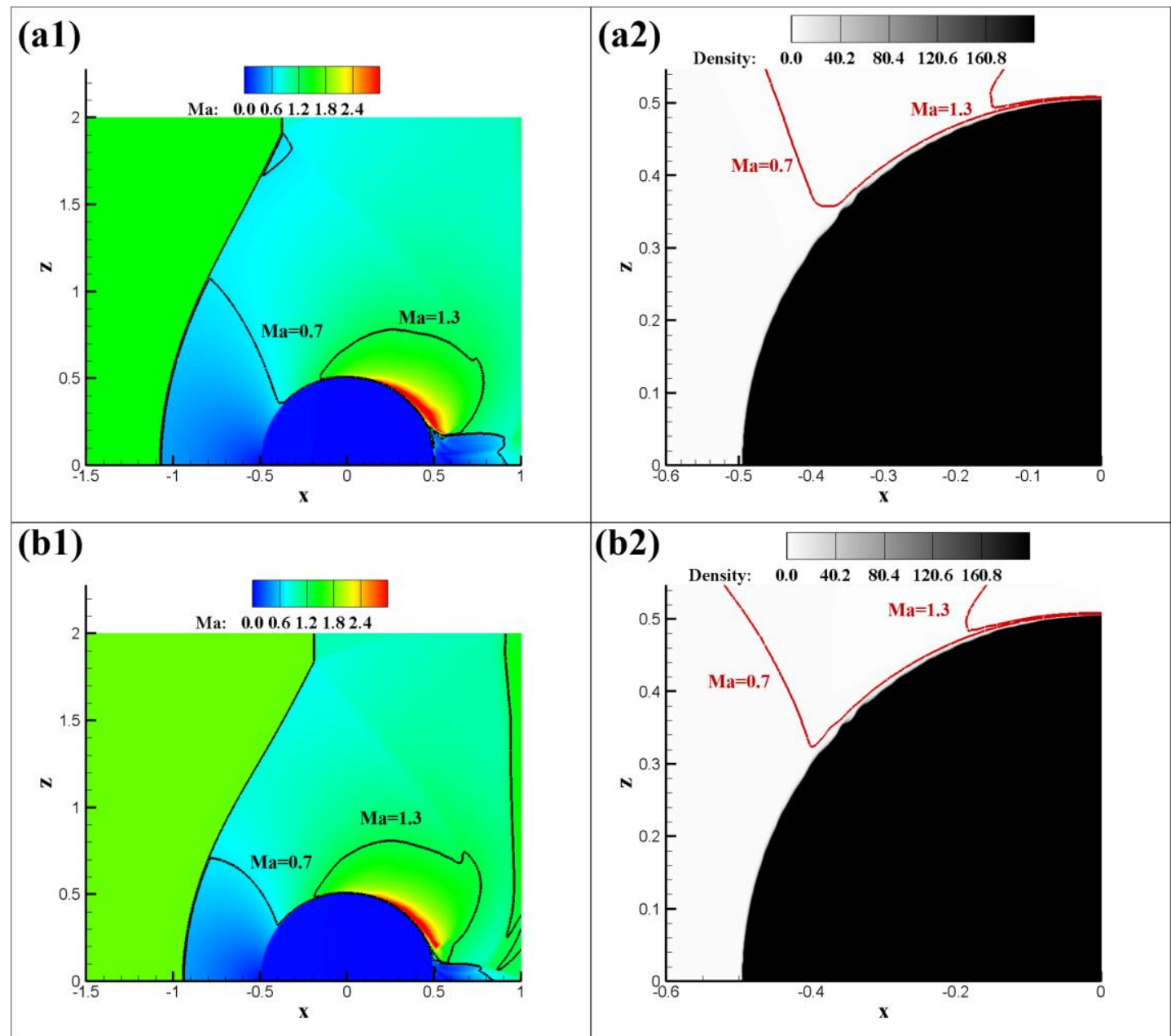


Figure $182 \mathrm{~d}$ simulations for cross-section deformation at $\mathrm{T}=\mathbf{0 . 0 6 4}$, (a)Ma

1.5 and (b) Ma 1.8, Ma contours and $\operatorname{density}\left(\mathrm{kg} / \mathrm{m}^{3}\right)$ contours

(a)

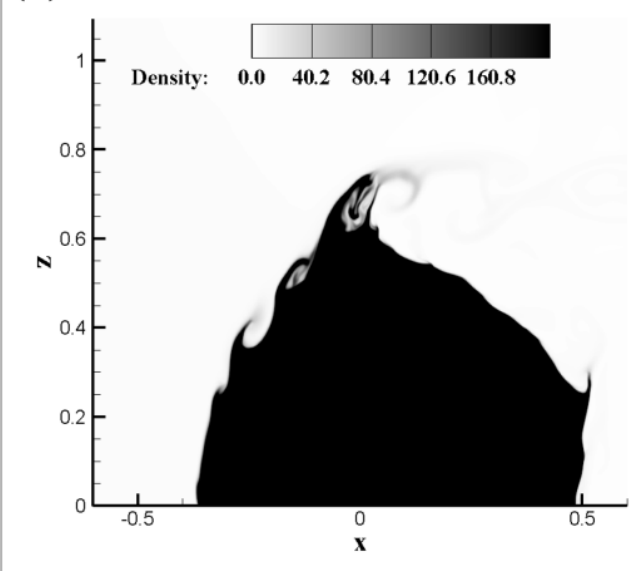

(b)

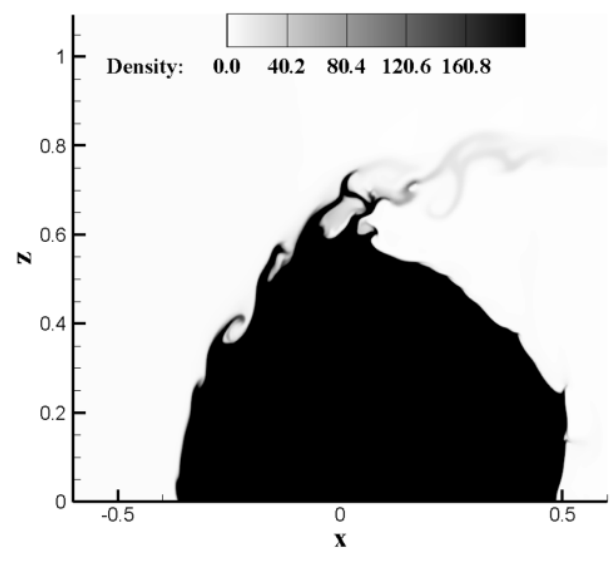

Figure $192 \mathrm{~d}$ simulations for cross-section deformation at $\mathrm{T}=\mathbf{0 . 3 8 6}$, (a)Ma

1.5 and (b) Ma 1.8, density $\left(\mathrm{kg} / \mathrm{m}^{3}\right)$ contours

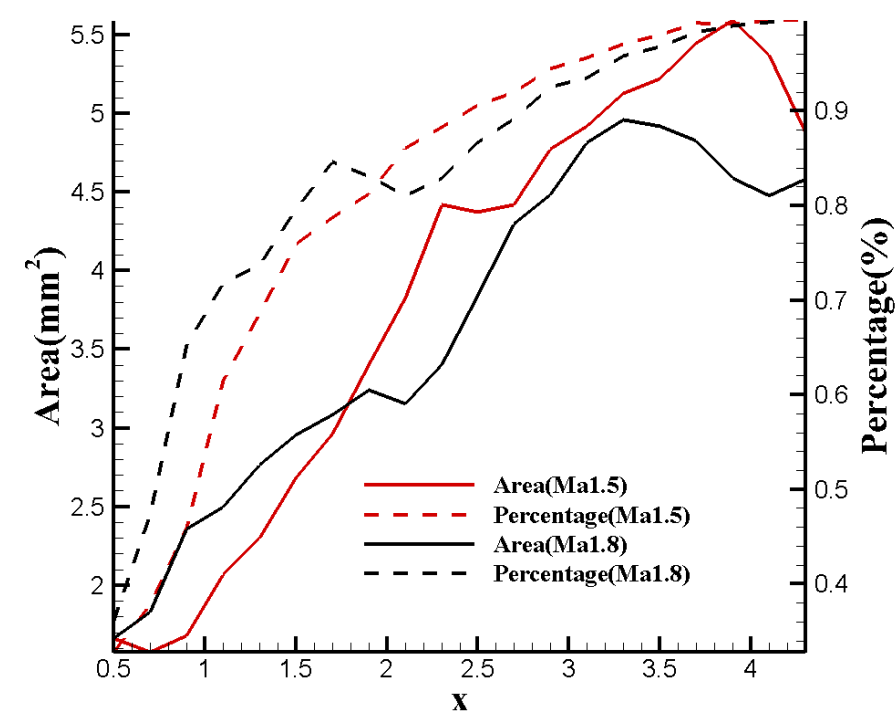

Figure 20 liquid areas on the slices of Y-Z plane along X-direction averaged over from $T=2.80$ to $T=3.65$, solid line: area of liquid( $\alpha>0)$, dashed line:

percentage of liquid under breakup $(0<\alpha<0.5)$ 


\subsection{Effect of the periodic injection}

Table 7 Periodic injection conditions

\begin{tabular}{llll}
\hline Injection velocity, $\mathrm{v}_{p l}(\mathrm{~m} / \mathrm{s})$ & $15.9[1.0+0.1 \sin (2 \pi \mathrm{ft})]$ & Frequency, $\mathrm{f}(\mathrm{kHz})$ & 100 \\
\hline
\end{tabular}

In the Table 7 , the velocity was set to vary sinusoidally in a high frequency. In such situation, the momentum ratio was calculated from the values averaged over one period time $\mathrm{T}_{\mathrm{p}}$ as the Equation (18) demonstrated. Besides, a Strouhal number of 6.29 in the current conditions was also obtained in a time averaged term which is showed in Equation (19). The momentum ratio directly related with the penetration ability is an obvious subject which has already been discussed in many works. And in this consideration, the influence of the momentum ratio was not concerned in this paper. As the Table 7 and Equation (18) illustrated, the amplitude of the velocity variation was relatively small comparing to the averaged velocity, and thus the momentum ratio of the periodic injection can be considered to be equal with the one in the previous sections.

$$
\begin{gathered}
q_{p}=\frac{\int_{0}^{T_{p}} \rho_{l} \mathrm{v}_{p l}^{2} \mathrm{~d} t / T_{p}}{\int_{0}^{T_{p}} \rho_{\infty} u_{\infty}^{2} d t / T_{p}}=1.005 q \approx q \\
\mathrm{Str}=f d / \int_{0}^{T_{p}} \mathrm{v}_{p l} d t / T_{p}
\end{gathered}
$$

The averaged momentum ratio of periodic injection conditions was almost the same with the momentum ratio of the pure liquid, thus there was no improvement on the penetration of the liquid jet under periodic injection conditions. Except no enhancement of the penetration height, the periodic injection imposed extra unstable waves on both windward and leeward side of the liquid surface as demonstrated in the Figure 21 and 22 where the instabilities generated in the pure liquid case were all 
covered by the imposed instabilities in the periodic injection condition. From the top view (Figure 22), the liquid film broke up at a position closer to the nozzle and the liquid after breakup distributed in a wider range in Z-direction. The column breakup process and the surface breakup process are compared in the Figure 23 and 24. The instabilities in the case of pure liquid mainly developed on the liquid surface, while in the case of the periodic injection the whole liquid film became wavy. The liquid near the wave crest and the liquid near the wave trough traveled in the opposite directions which would enhance the column breakup. As for the surface breakup displayed in the Figure 24 , the liquid was stripped in a wavy pattern and the unstable waves were augmented by the high speed crossflow. In general, the instabilities introduced by the periodic injection would have an effect on both the directions along and cross the injection. Following the quantitative analysis in the section 4.3 , we obtained the liquid distributions on Y-Z plane along X-direction in the Figure 25 in which the results from $\mathrm{t}=260 \mu$ s to $\mathrm{t}=320 \mu \mathrm{s}$ were averaged. It is apparent that the liquid jet with imposed instabilities experienced a greater breakup process and distributed in a wider range. The liquid area of pure liquid reaches peak at around $\mathrm{X}=3.1$ while periodically injected liquid at around $\mathrm{X}=2.4$. And then liquid areas of both of the two cases began to drop. This illustrates that the liquid of periodic injection broke up earlier than the pure liquid. 


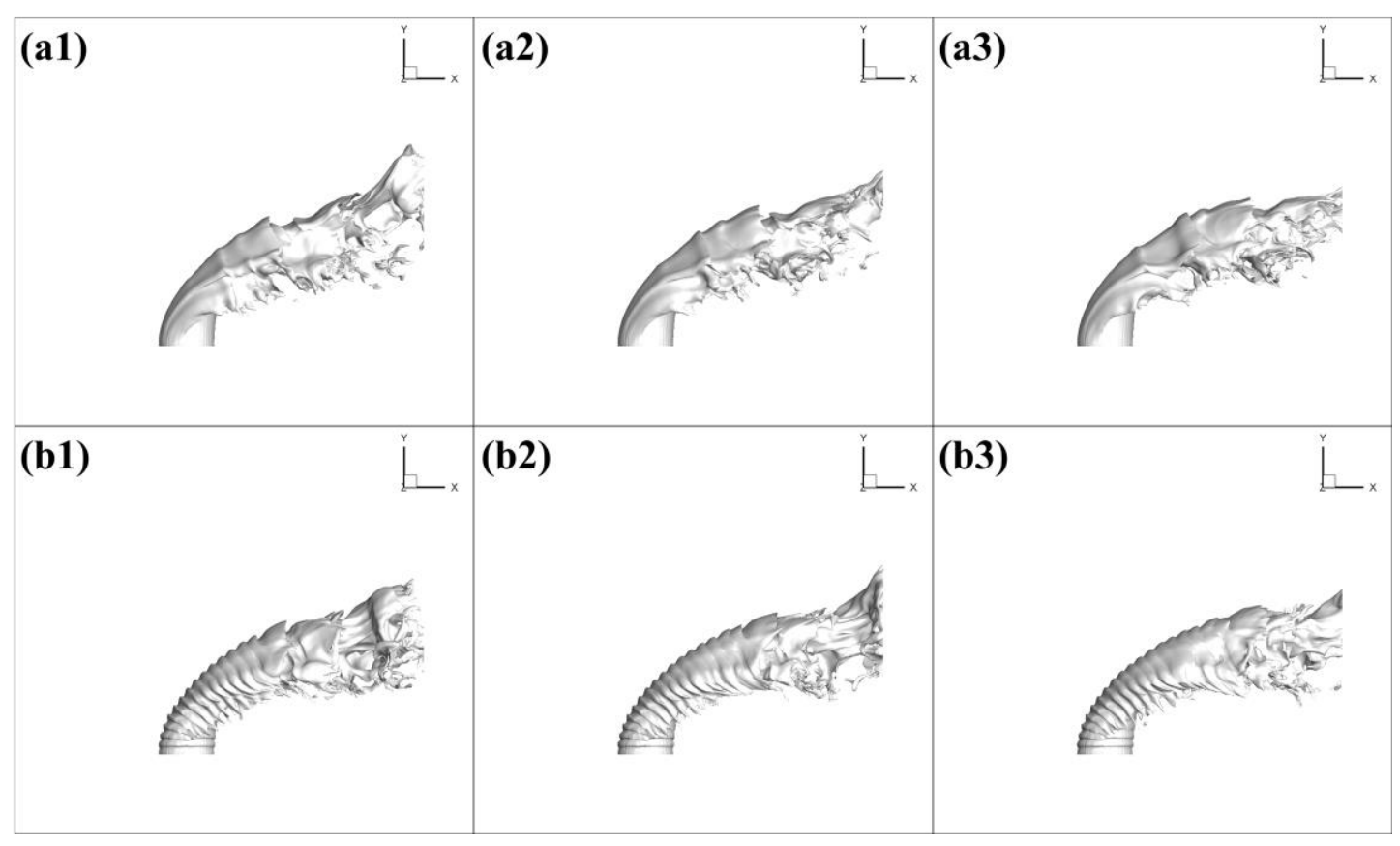

Figure 21 morphology comparison (side view) between cases of pure liquid and liquid injected periodically

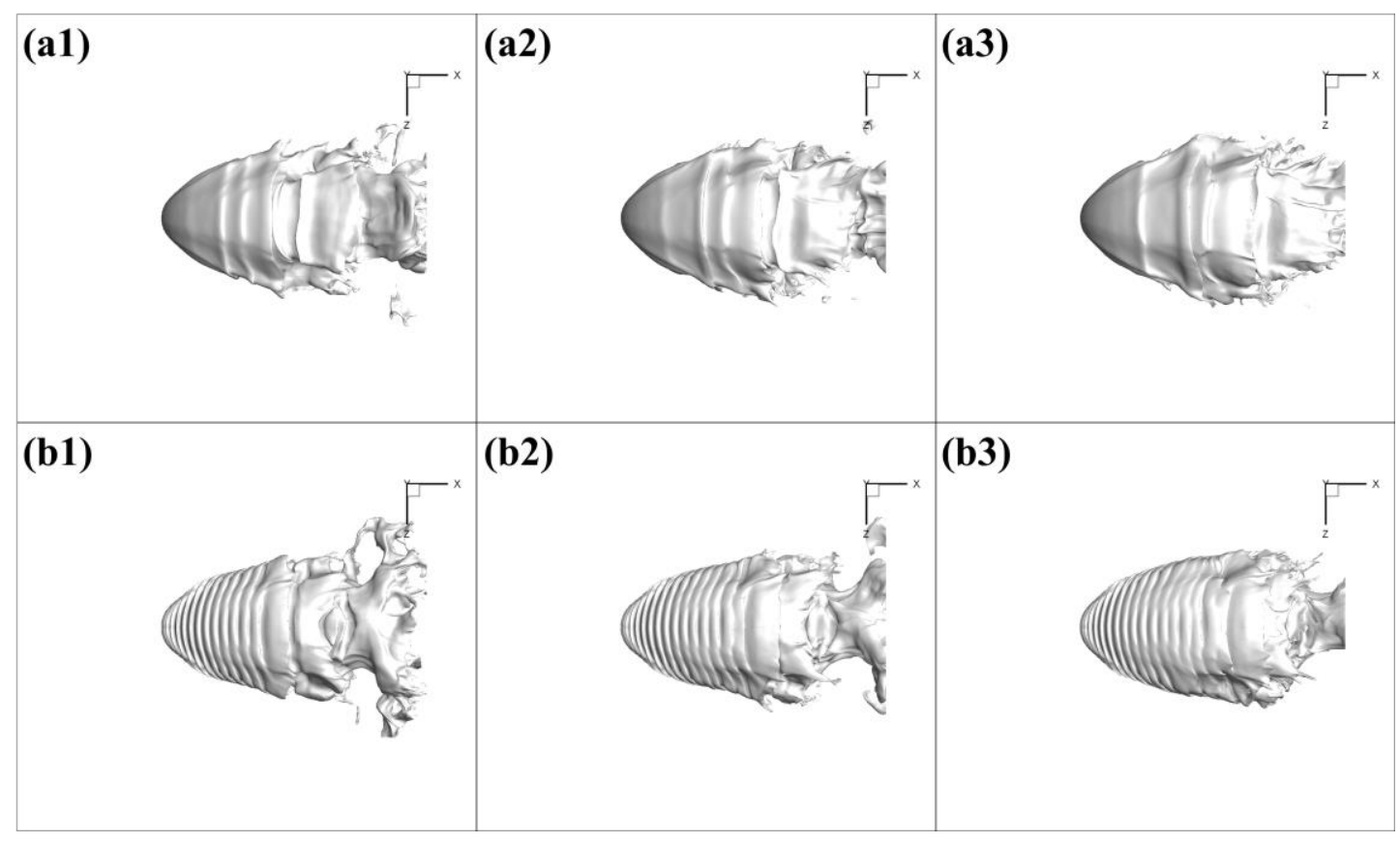

Figure 22 morphology comparison (top view) between cases of pure liquid and liquid injected periodically 

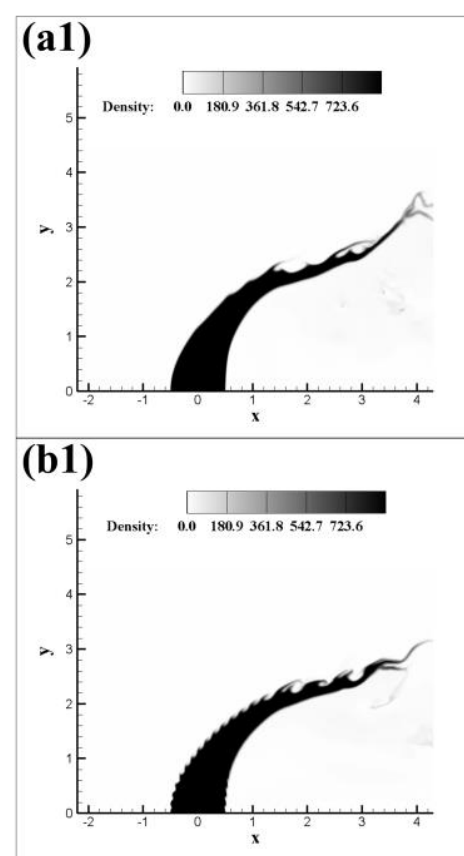

(a2)

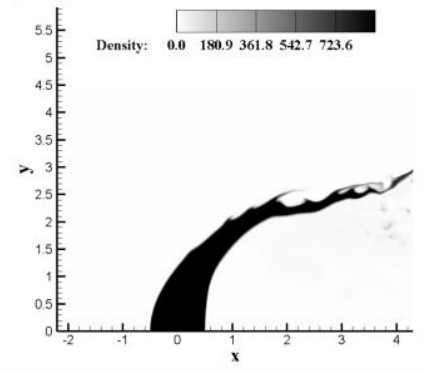

(b2)

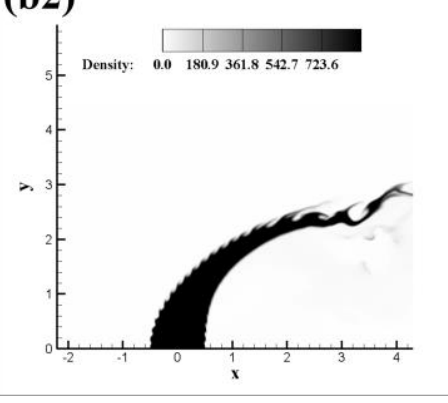

(a3)

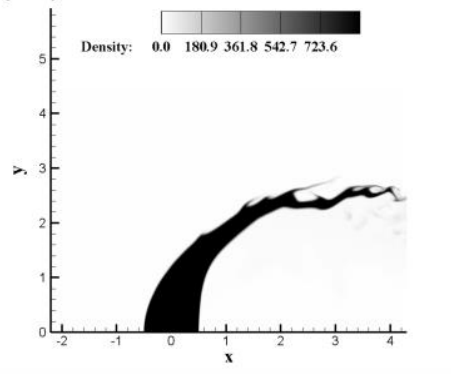

(b3)

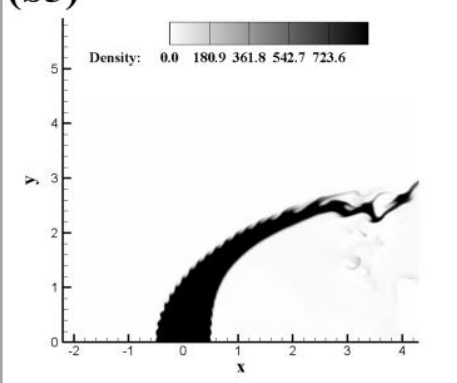

Figure 23 comparison of instabilities along injection direction between cases

(a)pure liquid and (b) liquid injected periodically, $\Delta \mathrm{t}=20 \mu \mathrm{s}$, density $\left(\mathrm{kg} / \mathrm{m}^{3}\right)$

contours
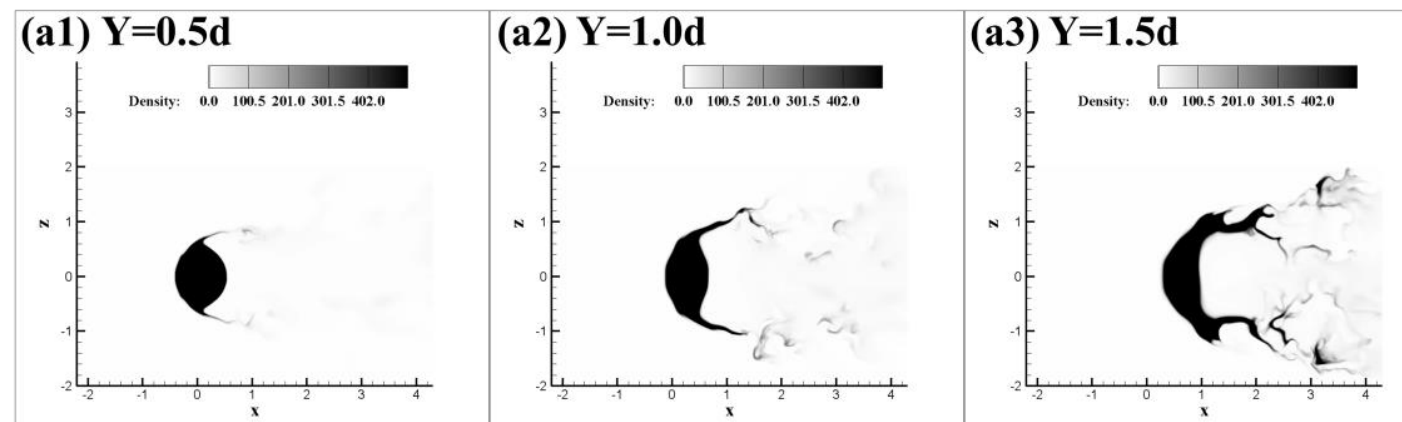

(b1) $Y=0.5 d$
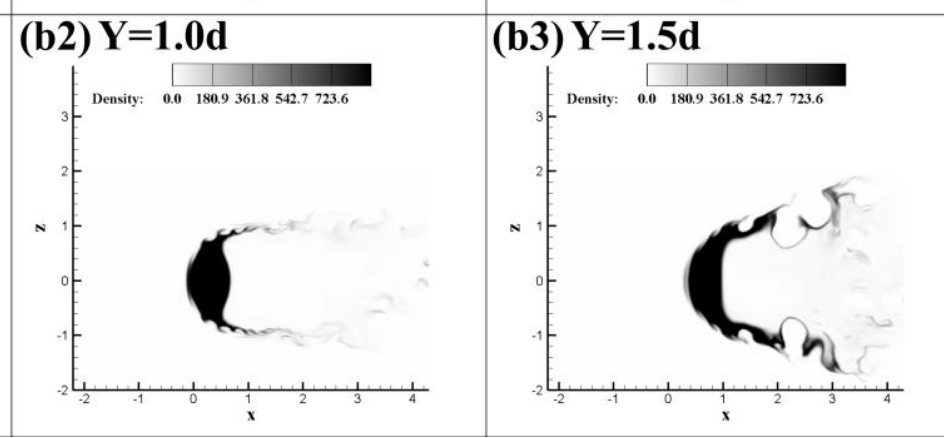

Figure 24 comparison of surface breakup process between cases (a)pure liquid and (b) liquid injected periodically at $\mathrm{t}=260 \mu \mathrm{s}$, density $\left(\mathrm{kg} / \mathrm{m}^{3}\right)$ contours 


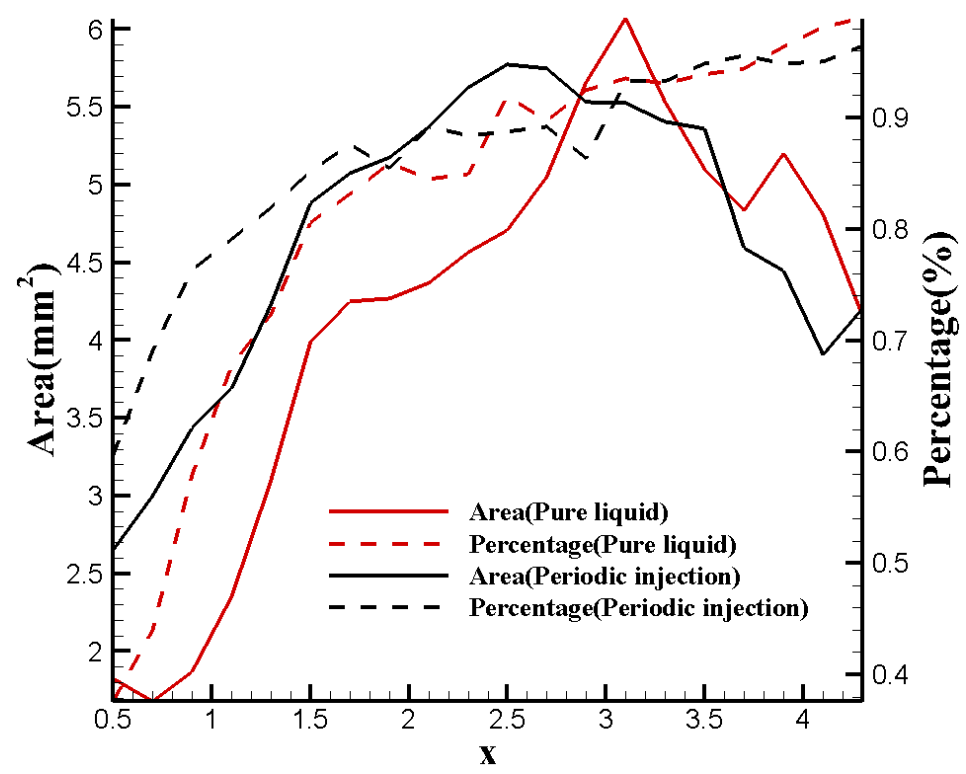

Figure 25 liquid areas on the slices of Y-Z plane along X-direction averaged over from $t=260 \mu$ s to $t=320 \mu$ s, solid line: area of liquid( $\alpha>0)$, dashed line: percentage of liquid under breakup $(0<\boldsymbol{\alpha}<0.5)$

\section{Conclusions}

The purpose of this paper was to establish a robust simulation system to study the liquid jet breakup in a supersonic crossflow. The compressible two-phase flows were simulated by the five-equation model and adaptive mesh refinement algorithm was adopted to balance the requirements of computational resources and space resolution. The following are some conclusions about the simulation system and the primary breakup of liquid jet in a supersonic crossflow.

(1) The Godunov method was combined with the wave propagation method to solve the two-phase system modeled by the five-equation model in order to apply the adaptive mesh refinement algorithm. The Godunov method was used to solve the density, momentum and energy equations while the wave propagation method was introduced 
to solve the advection equation which is non-conservative. The hybrid method was tailored to solve the five-equation system.

(2) A MOON-type positivity preserving method was applied in the high-order variable reconstruction to enhance the robustness of the simulation system. The positivity preserving method can detect the unphysical variables reconstructed by the high-order method. And these unphysical variables will be replaced by the variables reconstructed by a lower order method until the substitutive variables are feasible in the subsequent computational process.

(3) Primary breakup of pure liquid jet in a supersonic crossflow was studied based on the simulation results. The primary breakup process consists of two breakup modes. One is called column breakup and the other surface breakup. The development and traveling of the instable waves in two directions are responsible for the column and surface breakup respectively.

(4) The effect of the crossflow Mach number was studied by comparing the cases of Ma 1.5 and Ma 1.8. Crossflow of a higher Mach number was found being able to augment the instable waves along the injection direction which results in an easier column breakup. Besides, higher Mach flow also increased the number of the instabilities that is responsible for the surface breakup. The liquid jet expanded faster in the Ma 1.5 case than Ma 1.8 case because of the better carrying ability of the higher speed flows while the Ma 1.8 case reached a better atomization in the near-field.

(5) As the simulation results demonstrate, periodic injection of liquid reached no significant improvement of penetration while the periodic injection imposes extra 
instabilities on both windward and leeward sides. And the unstable waves introduced by the periodic conditions will improve the column breakup and surface breakup.

\section{Acknowledgements}

This work was supported by the National Natural Science Foundation of China (No. 11522222 and No. 91741205). The computation was performed using the computer facilities provided by the National Supercomputing Tianjin Center and National Supercomputing Changsha Center. And the development of simulation code in this work is in the framework of the open source software ARMOC.

\section{Reference}

[1] W. Huang, Transverse jet in supersonic crossflows, Aerospace Science and Technology, 50 (2016) 183-195.

[2] Z. Wang, L. Wu, Q. Li, C. Li, Experimental investigation on structures and velocity of liquid jets in a supersonic crossflow, Applied Physics Letters, 105 (2014) 2674-2686. [3] J. Huh, S. Lee, Numerical study on lateral jet interaction in supersonic crossflows, Aerospace Science and Technology, 80 (2018) 315-328.

[4] L.-q. Li, W. Huang, L. Yan, Z.-b. Du, M. Fang, Numerical investigation and optimization on the micro-ramp vortex generator within scramjet combustors with the transverse hydrogen jet, Aerospace Science and Technology, 84 (2019) 570-584.

[5] R. Feng, J. Li, Y. Wu, J. Zhu, X. Song, X. Li, Experimental investigation on gliding arc discharge plasma ignition and flame stabilization in scramjet combustor, Aerospace 
Science and Technology, 79 (2018) 145-153.

[6] J. Zhang, J. Chang, J. Ma, Y. Wang, W. Bao, Investigations on flame liftoff characteristics in liquid-kerosene fueled supersonic combustor equipped with thin strut, Aerospace Science and Technology, 84 (2019) 686-697.

[7] J. Zhang, J. Chang, J. Ma, Y. Zhang, W. Bao, Local and global flame characteristics in a liquid kerosene fueled supersonic combustor equipped with a thin strut, Aerospace Science and Technology, 76 (2018) 49-57.

[8] J. Zhang, J. Chang, J. Ma, C. Zhang, W. Bao, Investigation of flame establishment and stabilization mechanism in a kerosene fueled supersonic combustor equipped with a thin strut, Aerospace Science and Technology, 70 (2017) 152-160.

[9] L. Wu, Z.-g. Wang, Q. Li, C. Li, Study on transient structure characteristics of round liquid jet in supersonic crossflows Journal of Visualization, 19 (2016) 337-341.

[10] L. Wu, Z. Wang, Q. Li, J. Zhang, Investigations on the droplet distributions in the atomization of kerosene jets in supersonic crossflows, Applied Physics Letters, 107 (2015) 458-459.

[11] H. Sapmaz, B. Alkan, C.X. Lin, C. Ghenai, Visualization of Pulsed Aerated Liquid Jet in Supersonic Cross Flow, ASME 2005 Fluids Engineering Division Summer MeetingHouston, 2005.

[12] J.A. Schetz, E.A. Kush, P.B. Joshi, Wave phenomena in liquid jet breakup in a supersonic crossflow, AIAA Journal, 18 (1980) 774-778.

[13] K. Sallam, C. Aalburg, G.M. Faeth, Breakup of Round Nonturbulent Liquid Jets in Gaseous Crossflow AIAA Journal, 42 (2004) 2529-2540. 
[14] M. Arienti, M. C., Time-resolved proper orthogonal decomposition of liquid jet dynamics, Physics of Fluids, 21 (2009) 1979-1178.

[15] X. Li, M.C. Soteriou, Detailed numerical simulation of liquid jet atomization in crossflow of increasing density, International Journal of Multiphase Flow, 104 (2018) 214-232.

[16] S. Chen, D. Zhao, RANS investigation of the effect of pulsed fuel injection on scramjet HyShot II engine, Aerospace Science and Technology, 84 (2019) 182-192.

[17] V.B. Betelin, N.N. Smirnov, V.F. Nikitin, V.R. Dushin, A.G. Kushnirenko, V.A. Nerchenko, Evaporation and ignition of droplets in combustion chambers modeling and simulation, Acta Astronautica, 70 (2012) 23-35.

[18] P. Li, Z. Wang, M. Sun, H. Wang, Numerical simulation of the gas-liquid interaction of a liquid jet in supersonic crossflow, Acta Astronautica, 134 (2017) 333344.

[19] J. Glimm, X. Li, Y. Liu, N. Zhao, Conservative Front Tracking with Improved Accuracy, SIAM Journal on Numerical Analysis, 41 (2004) 1926-1947.

[20] C.W. Hirt, B.D. Nichols, Volume of fluid (VOF) method for the dynamics of free boundaries, Journal of Computational Physics, 39 (1981) 201-225.

[21] S. Osher, J.A. Sethian, Fronts propagating with curvature-dependent speed: Algorithms based on Hamilton-Jacobi formulations, Journal of Computational Physics, 79 (1988) 12-49.

[22] Y.H. Zhu, F. Xiao, Q.L. Li, R. Mo, C. Li, S. Lin, LES of primary breakup of pulsed liquid jet in supersonic crossflow, Acta Astronautica, 154 (2019) 119-132. 
[23] E. Johnsen, T. Colonius, Implementation of WENO schemes in compressible multicomponent flow problems, Journal of Computational Physics, 219 (2006) 715732.

[24] G. Allaire, S. Clerc, S. Kokh, A Five-Equation Model for the Simulation of Interfaces between Compressible Fluids, Journal of Computational Physics, 181 (2002) $577-616$.

[25] C.-H. Chang, M.-S. Liou, A robust and accurate approach to computing compressible multiphase flow: Stratified flow model and AUSM+-up scheme, Journal of Computational Physics, 225 (2007) 840-873.

[26] S.W. Ihm, C. Kim, Computations of Homogeneous-Equilibrium Two-Phase Flows with Accurate and Efficient Shock-Stable Schemes, AIAA Journal, 46 (2008) 30123037.

[27] K.M. Shyue, F. Xiao, An Eulerian interface sharpening algorithm for compressible two-phase flow: The algebraic THINC approach, Journal of Computational Physics, 268 (2014) 326-354.

[28] K.K. So, X.Y. Hu, N.A. Adams, Anti-diffusion Interface Sharpening Technique for Two-Phase Compressible Flow Simulations, Journal of Computational Physics, 231 (2012) 4304-4323.

[29] M.J. Berger, P. Colella, Local adaptive mesh refinement for shock hydrodynamics, Journal of Computational Physics, 82 (1989) 64-84.

[30] R. Deiterding, Detonation Structure Simulation with AMROC, International Conference on High Performance Computing \& Communications, Springer, Berlin, 
Heidelberg, 2005.

[31] R. Deiterding, An Adaptive Cartesian Detonation Solver for Fluid-Structure Interaction Simulation on Distributed Memory Computers, in: A. Deane, A. Ecer, J. McDonough, N. Satofuka, G. Brenner, D.R. Emerson, J. Periaux, D. Tromeur-Dervout (Eds.) Parallel Computational Fluid Dynamics 2005, Elsevier, Amsterdam, 2006, pp. 333-340.

[32] N. Liu, Z. Wang, M. Sun, H. Wang, B. Wang, Numerical simulation of liquid droplet breakup in supersonic flows, Acta Astronautica, 145 (2018) 116-130.

[33] J.C. Meng, T. Colonius, Numerical simulations of the early stages of high-speed droplet breakup, Shock Waves, 25 (2015) 399-414.

[34] A. Tiwari, J.B. Freund, C. Pantano, A Diffuse Interface Model with Immiscibility Preservation, J Comput Phys, 252 (2013) 290-309.

[35] R. Saurel, R.M. Abgrall, A Simple Method for Compressible Multifluid Flows, Siam Journal on Scientific Computing, 21 (1999) 1115-1145.

[36] G. Xiang, B. Wang, Numerical study of a planar shock interacting with a cylindrical water column embedded with an air cavity, Journal of Fluid Mechanics, 825 (2017) 825-852.

[37] K. Kitamura, Extension and Comparative Study of AUSM-Family Schemes for Compressible Multiphase Flow Simulations, Communications in Computational Physics, 16 (2014) 632-674.

[38] E.F. Toro, M. Spruce, W. Speares, Restoration of the contact surface in the HLLRiemann solver, Shock Waves, 4 (1994) 25-34. 
[39] R.J. LeVeque, Wave Propagation Algorithms for Multidimensional Hyperbolic Systems, Journal of Computational Physics, 131 (1997) 327-353.

[40] M.J. Berger, R.J. Leveque, Adaptive Mesh Refinement Using Wave-Propagation Algorithms for Hyperbolic Systems, SIAM Journal on Numerical Analysis, 35 (1998) 2298-2316.

[41] D.I. Ketcheson, M. Parsani, R.J. LeVeque, High-Order Wave Propagation Algorithms for Hyperbolic Systems, SIAM J. Sci. Comput., 35 (2013) A351-A377.

[42] B. Wang, G. Xiang, X.Y. Hu, An incremental-stencil WENO reconstruction for simulation of compressible two-phase flows, International Journal of Multiphase Flow 104 (2018) 20-31.

[43] X.Y. Hu, N. Adams, C.W. Shu, Positivity-preserving method for high-order conservative schemes solving compressible Euler equations, Journal of Computational Physics, 242 (2013) 169-180.

[44] S. Sembian, M. Liverts, N. Tillmark, N. Apazidis, Plane shock wave interaction with a cylindrical water column, Physics of Fluids, 28 (2016) 741-757.

[45] T.G. Theofanous, V.V. Mitkin, C.L. Ng, C. Chang, X. Deng, S. Sushchikh, The physics of aerobreakup. II. Viscous liquids, Physics of Fluids, 24 (2012) 052103052180.

[46] G.E.A. Meier, A.P. Szumowski, W.C. Selerowicz, Self-excited oscillations in internal transonic flows, Progress in Aerospace Sciences, 27 (1990) 145-200. 